\title{
ROMPgel-supported Thiazolium lodide: An Efficient Supported Organic Catalyst For Parallel Stetter Reactions
}

\author{
Anthony G. M. Barrett, ${ }^{*}$ Andrew C. Love and Livio Tedeschi \\ Department of Chemistry, Imperial College London, Exhibition Road, London SW7 2AZ, England \\ agmb@imperial.ac.uk
}

Supporting Information 1 of 2

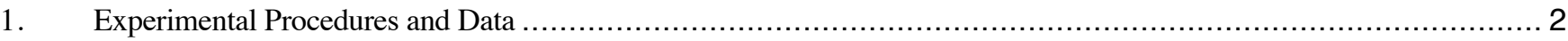

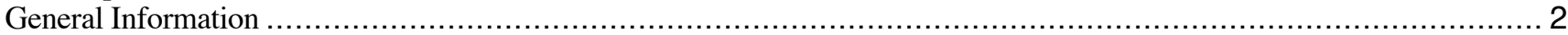

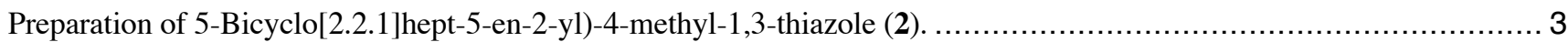

Preparation of 5-endo-Bicyclo[2.2.1] hept-5-en-2-yl)-3,4-dimethyl-1,3-thiazol-3-ium Iodide (3a) ......................... 3

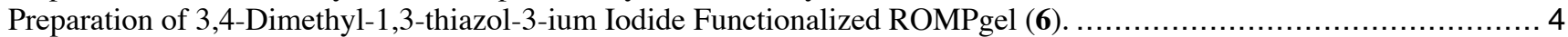

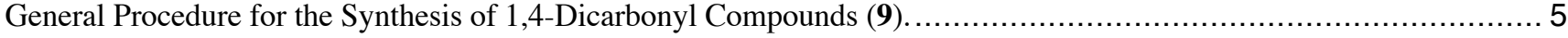

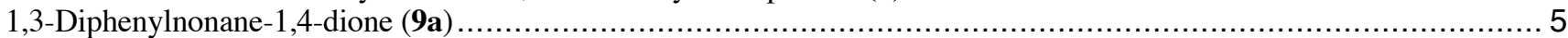

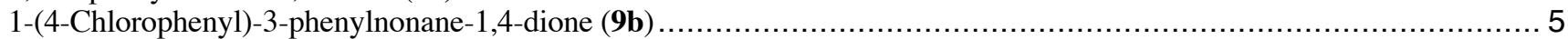

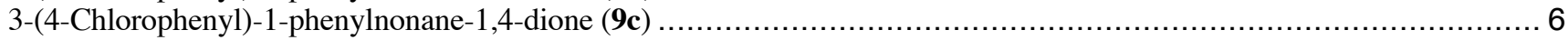

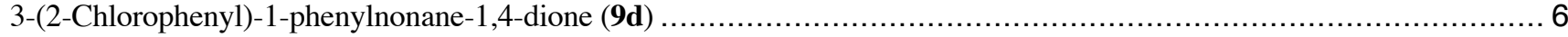

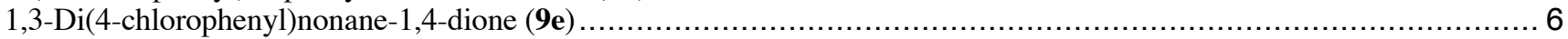

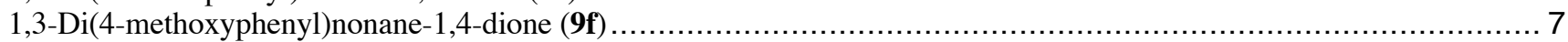

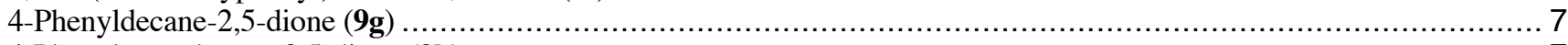

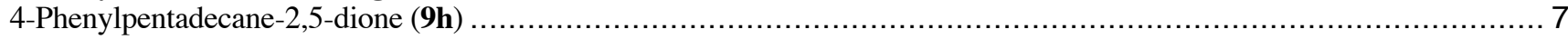

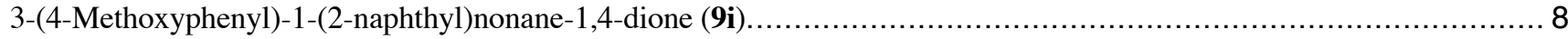

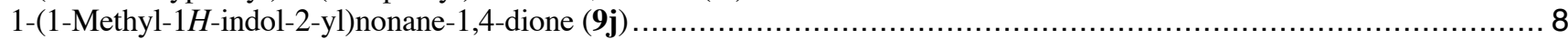

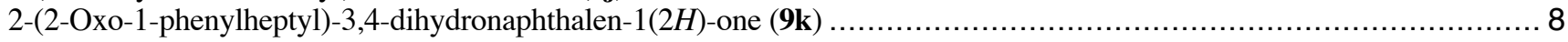

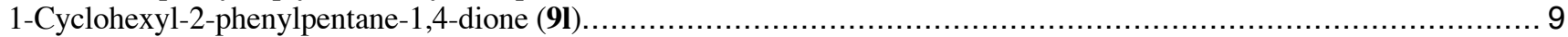

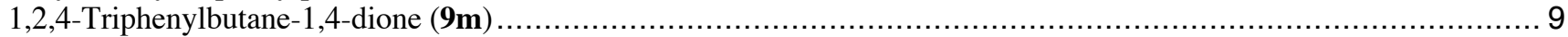

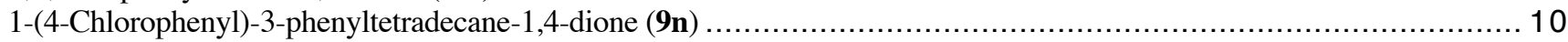

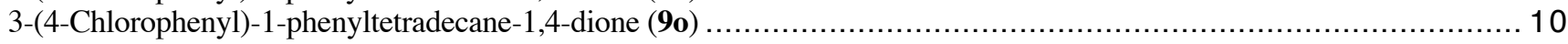

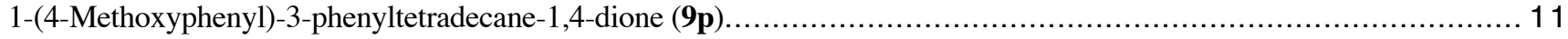

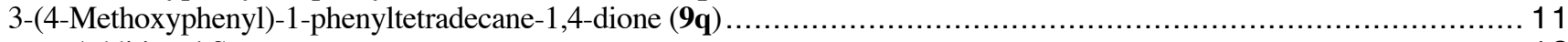

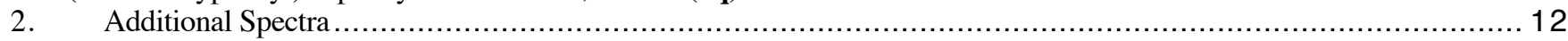




\section{Experimental Procedures and Data}

\section{General Information}

All reagents were obtained from commercial suppliers and used without further purification unless otherwise stated. Where solvents have been described they had been vigorously dried over $\mathrm{CaH}_{2}\left(\mathrm{CH}_{2} \mathrm{Cl}_{2}\right)$, $\mathrm{Na} / \mathrm{Ph}_{2} \mathrm{CO}\left(\mathrm{Et}_{2} \mathrm{O}\right), \mathrm{Mg}(\mathrm{MeOH})$ or $\mathrm{K} / \mathrm{Ph}_{2} \mathrm{CO}(\mathrm{THF})$. All reactions were carried out in flame-dried or ovendried glassware under a dry nitrogen atmosphere at ambient temperature unless otherwise stated. Flash column chromatography was carried out on BDH silica gel 60, grade 40-63 $\mu \mathrm{m}$. Reactions were monitored by thin layer chromatography (TLC) using Merck Kieselgel $60 \mathrm{~F}_{254}$ aluminum sheets. Visualization was effected with short wavelength UV light $(254 \mathrm{~nm})$ or chemical staining using either a solution of basic $\mathrm{KMnO}_{4}$ or a solution of vanillin and concentrated sulfuric acid in ethanol. 


\section{Preparation of 5-Bicyclo[2.2.1]hept-5-en-2-yl)-4-methyl-1,3-thiazole (2).}
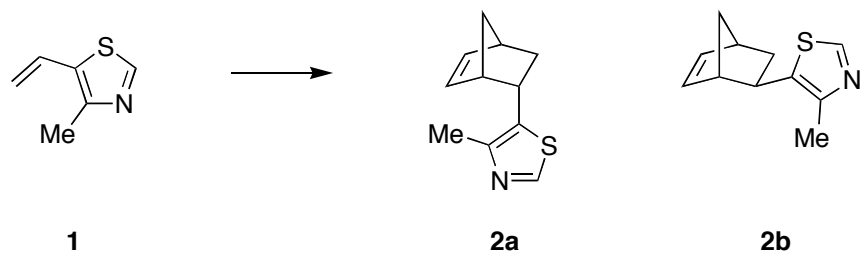

Cyclopentadiene $(5.77 \mathrm{~g}, 87.3 \mathrm{mmol})$ was added to $1(1 \mathrm{~mL}, 8.73 \mathrm{mmol})$ and the reaction mixture heated to $200{ }^{\circ} \mathrm{C}$ for $1 \mathrm{~h}, 230{ }^{\circ} \mathrm{C}$ for $0.5 \mathrm{~h}$ and $200{ }^{\circ} \mathrm{C}$ for $1.5 \mathrm{~h}$. The mixture was cooled to $24{ }^{\circ} \mathrm{C}$ and concentrated in vacuo. Flash column chromatography (30 : 70; EtOAc : hexanes) gave 5-bicyclo[2.2.1]hept-5-en-2-yl)4-methyl-1,3-thiazole (2) (1.19 g, 53\% yield) a clear colorless oil, as a mixture of (1:9.5) exo : endo isomers: $R_{f} 0.38$ (EtOAc : hexanes $2: 3$ ); IR (thin film) 1532, 1447, 1416, 1376, 1340, 1247, 925, 844, 785, $730 \mathrm{~cm}^{-1}$; 5-endo-bicyclo[2.2.1]hept-5-en-2-yl)-4-methyl-1,3-thiazole (2a) ${ }^{1} \mathrm{H}$ NMR $\left(500 \mathrm{MHz}, \mathrm{CDCl}_{3}\right) \delta$ $8.45(\mathrm{~s}, 1 \mathrm{H}), 6.35(\mathrm{dd}, J=5.6 \mathrm{~Hz}, J=3.0 \mathrm{~Hz}, 1 \mathrm{H}), 5.91(\mathrm{dd}, J=5.6 \mathrm{~Hz}, J=3.0 \mathrm{~Hz}, 1 \mathrm{H}), 3.57(\mathrm{~m}, 1 \mathrm{H})$, $3.02(\mathrm{~s}, 1 \mathrm{H}), 2.96(\mathrm{~s}, 1 \mathrm{H}), 2.45(\mathrm{~s}, 3 \mathrm{H}), 2.40-2.33(\mathrm{~m}, 1 \mathrm{H}), 1.58-1.55(\mathrm{~m}, 1 \mathrm{H}), 1.47-1.45(\mathrm{~m}, 1 \mathrm{H}), 1.02$ $(\mathrm{ddd}, J=11.9 \mathrm{~Hz}, J=4.5 \mathrm{~Hz}, J=2.7 \mathrm{~Hz}, 1 \mathrm{H}) ;{ }^{13} \mathrm{C} \mathrm{NMR}\left(126 \mathrm{MHz}, \mathrm{CDCl}_{3}\right) \delta 148.5,148.0,138.9,136.9$, 132.8, 50.3 , 47.9, 43.3, 37.7, 36.2, 15.3; 5-exo-bicyclo[2.2.1] hept-5-en-2-yl)-4-methyl-1,3-thiazole $(\mathbf{2 b}){ }^{1} \mathrm{H}$ $\operatorname{NMR}\left(500 \mathrm{MHz}, \mathrm{CDCl}_{3}\right) \delta 8.52(\mathrm{~s}, 1 \mathrm{H}), 6.24(\mathrm{dd}, J=5.6 \mathrm{~Hz}, J=3.0 \mathrm{~Hz}, 1 \mathrm{H}), 6.15(\mathrm{dd}, J=5.6 \mathrm{~Hz}, J=$ $3.0 \mathrm{~Hz}, 1 \mathrm{H}), 3.00(\mathrm{~s}, 1 \mathrm{H}), 2.86(\mathrm{dd}, J=8.3 \mathrm{~Hz}, J=4.4 \mathrm{~Hz}, 1 \mathrm{H}), 2.79(\mathrm{~s}, 1 \mathrm{H}), 2.40(\mathrm{~s}, 3 \mathrm{H}), 1.81$ - $1.76(\mathrm{~m}$, $1 \mathrm{H}), 1.65-1.63(\mathrm{~m}, 1 \mathrm{H}), 1.61-1.50(\mathrm{~m}, 1 \mathrm{H}), 1.46(\mathrm{~m}, 1 \mathrm{H}) ;{ }^{13} \mathrm{C} \mathrm{NMR}\left(126 \mathrm{MHz}, \mathrm{CDCl}_{3}\right) \delta 148.2,148.0$, 137.4, 137.0, 136.6, 49.4, 46.2, 42.2, 36.5, 36.4, 15.3; MS (CI, $\left.\mathrm{NH}_{3}\right) \mathrm{m} / z 192(\mathrm{M}+\mathrm{H})^{+}$; HRMS $\left(\mathrm{CI}, \mathrm{NH}_{3}\right)$ $m / z$ calc for $\mathrm{C}_{11} \mathrm{H}_{14} \mathrm{NS}: 192.0847$; found: 192.0841. Anal. calc for $\mathrm{C}_{11} \mathrm{H}_{13} \mathrm{NS}$ : C, 69.07; H, 6.85; N, 7.32. Found: C, 68.93; H, 6.74; N, 7.21.

\section{Preparation of 5-endo-Bicyclo[2.2.1]hept-5-en-2-yl)-3,4-dimethyl-1,3-thiazol-3-ium Iodide (3a).}

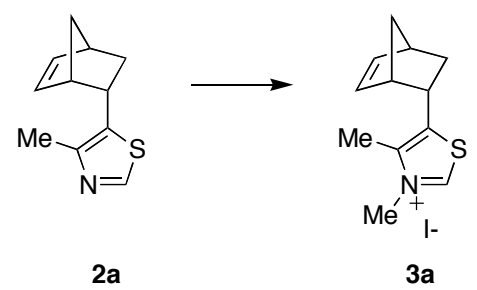

MeI (0.05 mL, $0.74 \mathrm{mmol})$ was added to $\mathbf{2 a}(94.4 \mathrm{mg}, 0.49 \mathrm{mmol})$ in $n$-BuOH $(2.5 \mathrm{~mL})$ and the mixture heated to $70{ }^{\circ} \mathrm{C}$. After $58 \mathrm{~h}$, the reaction mixture was concentrated in vacuo. The crude solid was recrystallized $\left(\mathrm{CH}_{2} \mathrm{Cl}_{2}: \mathrm{Et}_{2} \mathrm{O}\right)$ to give 5-endo-bicyclo[2.2.1]hept-5-en-2-yl)-3,4-dimethyl-1,3-thiazol-3-ium iodide 3a (143.3 mg, 87\%) as yellow crystals: mp $155-160{ }^{\circ} \mathrm{C}$; IR (solid) $1591,1479,1336,738 \mathrm{~cm}^{-1} ;{ }^{1} \mathrm{H}$ NMR $\left(500 \mathrm{MHz}, \mathrm{CDCl}_{3}\right) \delta 10.8(\mathrm{~s}, 1 \mathrm{H}), 6.53(\mathrm{~m}, 1 \mathrm{H}), 6.01(\mathrm{~m}, 1 \mathrm{H}), 4.38(\mathrm{~m}, 3 \mathrm{H}), 3.68(\mathrm{~m}, 1 \mathrm{H}), 3.11(\mathrm{~s}$, $2 \mathrm{H}), 2.61(\mathrm{~s}, 3 \mathrm{H}), 2.55-2.46(\mathrm{~m}, 1 \mathrm{H}), 1.71-1.68(\mathrm{~m}, 1 \mathrm{H}), 1.56-1.54(\mathrm{~m}, 1 \mathrm{H}), 1.14-1.09(\mathrm{~m}, 1 \mathrm{H}) ;{ }^{13} \mathrm{C}$ NMR $\left(126 \mathrm{MHz}, \mathrm{CDCl}_{3}\right) \delta 155.6,143.7,141.0,140.9,132.3,50.7,48.0,43.4,41.7,37.9,37.1,12.7$; MS $(\mathrm{FAB}+) \mathrm{m} / z 206(\mathrm{M}-\mathrm{I})^{+}$; HRMS $(\mathrm{FAB}+) \mathrm{m} / z$ calc for $\mathrm{C}_{12} \mathrm{H}_{16} \mathrm{NS}: 206.1003$; found: 206.1013. Anal. calc for $\mathrm{C}_{12} \mathrm{H}_{16} \mathrm{INS}$ : C, 43.25; H, 4.84; N, 4.20. Found: C, 43.30; H, 4.71; N, 4.13. 
Preparation of 3,4-Dimethyl-1,3-thiazol-3-ium Iodide Functionalized ROMPgel (6).

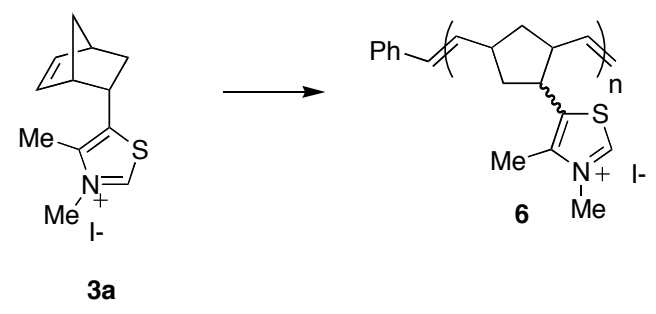

1,3-Dimesityl-4,5-dihydroimidazol-2-ylidene ruthenium benzylidene (5) $(12.0 \mathrm{mg}, 0.01 \mathrm{mmol})$ in $\mathrm{ClCH}_{2} \mathrm{CH}_{2} \mathrm{Cl}(0.25 \mathrm{~mL})$ was added to $3 \mathbf{a}(426.3 \mathrm{mg}, 1.28 \mathrm{mmol})$ and cross-linker $4(37.3 \mathrm{mg}, 0.14 \mathrm{mmol})$ in $\mathrm{ClCH}_{2} \mathrm{CH}_{2} \mathrm{Cl}(2 \mathrm{~mL})$ at $50{ }^{\circ} \mathrm{C}$. After $16 \mathrm{~h} \mathrm{CH}_{3} \mathrm{CN}(2 \mathrm{~mL})$ and $\mathrm{Et}_{2} \mathrm{O}(2 \mathrm{~mL})$ were added and the reaction mixture was heated to $50^{\circ} \mathrm{C}$ for $2 \mathrm{~h}$. The ROMPgel was filtered off and rinsed repeatedly with alternating $\mathrm{Et}_{2} \mathrm{O}(6 \times 10 \mathrm{~mL})$ and $\mathrm{CH}_{2} \mathrm{Cl}_{2}(6 \times 10 \mathrm{~mL})$. The ROMPgel was dried under high vacuum to a yellow ROMPgel 6 (94\% yield): IR (solid) 1456, 975, $923 \mathrm{~cm}^{-1}$. Anal. Found: C, 48.57; H, 5.23; N, 3.54. 


\section{General Procedure for the Synthesis of 1,4-Dicarbonyl Compounds (9).}

In a typical experiment, DMF ( $2 \mathrm{~mL})$ was added to the $\alpha, \beta$-unsaturated ketone 8 (222 $\mathrm{mg}, 0.92 \mathrm{mmol})$ and 3,4-dimethyl-1,3-thiazol-3-ium iodide functionalized ROMPgel (6) (59.3 mg, $0.15 \mathrm{mmol}$ ). Aldehyde 7c $(0.11 \mathrm{~mL}, 0.92 \mathrm{mmol})$ was added to the reaction mixture, followed by $\mathrm{Et}_{3} \mathrm{~N}(1.02 \mathrm{~mL}, 7.33 \mathrm{mmol})$ and the reaction heated at $80{ }^{\circ} \mathrm{C}$ for $22 \mathrm{~h}$. When the transformation was complete (GC-MS), the mixture was concentrated in vacuo and filtered through a plug of silica with hexanes. Concentration of the filtrate gave the corresponding 1,4-dicarbonyl compound 9.

\section{1,3-Diphenylnonane-1,4-dione $(9 a)^{1}$}

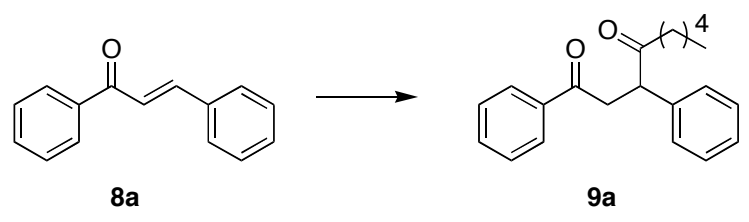

1,3-Diphenylnonane-1,4-dione (121 mg, 89\% yield, 90\% purity) was obtained as a yellow oil: $R_{f} 0.73$ $\left(\mathrm{Et}_{2} \mathrm{O}\right)$; IR (thin film) 1713, 1684, 1449, 752, 702, $690 \mathrm{~cm}^{-1}$; ${ }^{1} \mathrm{H}$ NMR $\left(300 \mathrm{MHz}, \mathrm{CDCl}_{3}\right) \delta 7.97$ - 7.95 (m, $2 \mathrm{H}), 7.57-7.26(\mathrm{~m}, 8 \mathrm{H}), 4.42(\mathrm{dd}, J=10.2 \mathrm{~Hz}, J=3.6 \mathrm{~Hz}, 1 \mathrm{H}), 4.04(\mathrm{dd}, J=18.0 \mathrm{~Hz}, J=10.2 \mathrm{~Hz}, 1 \mathrm{H})$, $3.12(\mathrm{dd}, J=18.0 \mathrm{~Hz}, J=3.6 \mathrm{~Hz}, 1 \mathrm{H}), 2.65-2.57(\mathrm{~m}, 1 \mathrm{H}), 2.51-2.43(\mathrm{~m}, 1 \mathrm{H}), 1.68-1.49(\mathrm{~m}, 2 \mathrm{H}), 1.31$ - $1.13(\mathrm{~m}, 4 \mathrm{H}), 0.83(\mathrm{t}, J=7.1 \mathrm{~Hz}, 3 \mathrm{H}) ;{ }^{13} \mathrm{C} \mathrm{NMR}\left(75 \mathrm{MHz}, \mathrm{CDCl}_{3}\right) \delta$ 209.5, 198.2, 138.2, 136.5, 133.2, 129.1, 128.5, 128.3, 128.1, 127.5, 53.3, 42.3, 41.8, 31.1, 23.3, 22.5, 14.0; MS (CI, $\left.\mathrm{NH}_{3}\right) \mathrm{m} / z 326$ $\left(\mathrm{M}+\mathrm{NH}_{4}\right)^{+}, 309(\mathrm{M}+\mathrm{H})^{+} ; \mathrm{HRMS}\left(\mathrm{CI}, \mathrm{NH}_{3}\right) \mathrm{m} / z$ calc for $\mathrm{C}_{21} \mathrm{H}_{25} \mathrm{O}_{2}: 309.1855$; found: 309.1862 .

\section{1-(4-Chlorophenyl)-3-phenylnonane-1,4-dione (9b)}

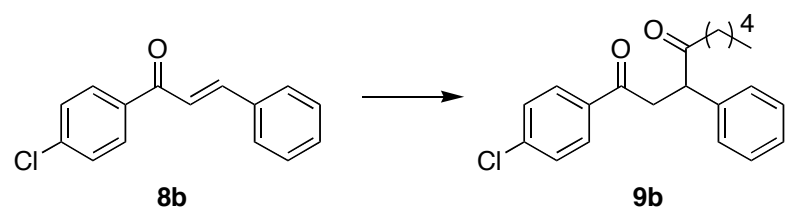

1-(4-Chlorophenyl)-3-phenylnonane-1,4-dione (342 mg, 92\% yield, 95\% purity) was obtained as a orange oil: $R_{f} 0.71\left(\mathrm{Et}_{2} \mathrm{O}\right)$; IR (thin film) 1713, 1682, 1590, 1401, 1091, $700 \mathrm{~cm}^{-1} ;{ }^{1} \mathrm{H}$ NMR $\left(300 \mathrm{MHz}, \mathrm{CDCl}_{3}\right) \delta$ $7.86(\mathrm{~d}, J=8.5 \mathrm{~Hz}, 2 \mathrm{H}), 7.37-7.26(\mathrm{~m}, 7 \mathrm{H}), 4.41(\mathrm{dd}, J=10.1 \mathrm{~Hz}, J=3.3 \mathrm{~Hz}, 1 \mathrm{H}), 3.99(\mathrm{dd}, J=18.0 \mathrm{~Hz}$, $J=10.1 \mathrm{~Hz}, 1 \mathrm{H}), 3.04(\mathrm{dd}, J=18.0 \mathrm{~Hz}, J=3.3 \mathrm{~Hz}, 1 \mathrm{H}), 2.64-2.39(\mathrm{~m}, 2 \mathrm{H}), 1.60-1.46(\mathrm{~m}, 2 \mathrm{H}), 1.34-$ $1.17(\mathrm{~m}, 4 \mathrm{H}), 0.81(\mathrm{t}, J=6.8 \mathrm{~Hz}, 3 \mathrm{H}) ;{ }^{13} \mathrm{C} \mathrm{NMR}\left(75 \mathrm{MHz}, \mathrm{CDCl}_{3}\right) \delta 209.1,196.9,139.5,138.1,134.9$, $129.5,129.1,128.8,128.3,127.6,53.3,42.3,41.7,31.2,23.3,22.4,13.9 ; \mathrm{MS}\left(\mathrm{CI}, \mathrm{NH}_{3}\right) \mathrm{m} / z 368$ $\left(\mathrm{M}+\mathrm{NH}_{4}\right)^{+}, 343(\mathrm{M}+\mathrm{H})^{+}$; HRMS $\left(\mathrm{CI}, \mathrm{NH}_{3}\right) \mathrm{m} / z$ calc for $\mathrm{C}_{21} \mathrm{H}_{24} \mathrm{O}_{2} \mathrm{Cl}$ : 343.1465; found: 343.1465. 


\section{3-(4-Chlorophenyl)-1-phenylnonane-1,4-dione (9c)}

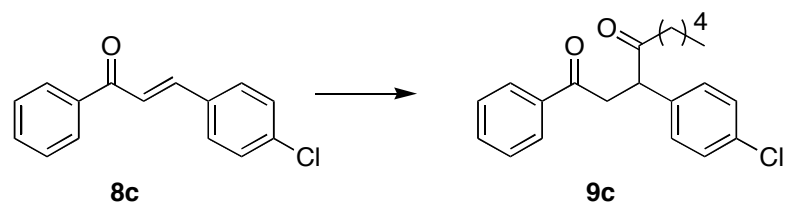

3-(4-Chlorophenyl)-1-phenylnonane-1,4-dione (308 mg, 98\% yield, 95\% purity) was obtained as a yellow oil: $R_{f} 0.71\left(\mathrm{Et}_{2} \mathrm{O}\right)$; IR (thin film) 1713, 1682, 1489, 1015, 754, $689 \mathrm{~cm}^{-1} ;{ }^{1} \mathrm{H}$ NMR $\left(300 \mathrm{MHz}, \mathrm{CDCl}_{3}\right) \delta$ $7.94(\mathrm{~d}, J=7.2 \mathrm{~Hz}, 2 \mathrm{H}), 7.55-7.50(\mathrm{~m}, 1 \mathrm{H}), 7.44-7.39(\mathrm{~m}, 2 \mathrm{H}), 7.31(\mathrm{~d}, J=8.4 \mathrm{~Hz}, 2 \mathrm{H}), 7.23(\mathrm{~d}, J=8.4$ $\mathrm{Hz}, 2 \mathrm{H}), 4.40(\mathrm{dd}, J=9.9 \mathrm{~Hz}, J=3.6 \mathrm{~Hz}, 1 \mathrm{H}), 4.00(\mathrm{dd}, J=18.0 \mathrm{~Hz}, J=9.9 \mathrm{~Hz}, 1 \mathrm{H}), 3.11(\mathrm{dd}, J=18.0$ $\mathrm{Hz}, J=3.6 \mathrm{~Hz}, 1 \mathrm{H}), 2.68-2.40(\mathrm{~m}, 2 \mathrm{H}), 1.61-1.45(\mathrm{~m}, 2 \mathrm{H}), 1.35-1.15(\mathrm{~m}, 4 \mathrm{H}), 0.83(\mathrm{t}, J=6.9 \mathrm{~Hz}, 3 \mathrm{H})$; ${ }^{13} \mathrm{C}$ NMR $\left(75 \mathrm{MHz}, \mathrm{CDCl}_{3}\right) \delta$ 209.0, 197.8, 136.8, 136.4, 133.5, 133.3, 129.7, 129.2, 128.6, 128.1, 52.6, 42.3, 41.9, 31.2, 23.3, 22.4, 13.9; $\mathrm{MS}\left(\mathrm{CI}, \mathrm{NH}_{3}\right) \mathrm{m} / \mathrm{z} 360\left(\mathrm{M}+\mathrm{NH}_{4}\right)^{+}, 343(\mathrm{M}+\mathrm{H})^{+}$; HRMS (CI, $\left.\mathrm{NH}_{3}\right) \mathrm{m} / z$ calc for $\mathrm{C}_{21} \mathrm{H}_{24} \mathrm{O}_{2} \mathrm{Cl}$ : 343.1465; found: 343.1460. Anal. calc for $\mathrm{C}_{21} \mathrm{H}_{23} \mathrm{O}_{2} \mathrm{Cl}: \mathrm{C}, 73.57 ; \mathrm{H}, 6.76$. Found: C, 73.36; H, 6.87.

\section{3-(2-Chlorophenyl)-1-phenylnonane-1,4-dione (9d)}

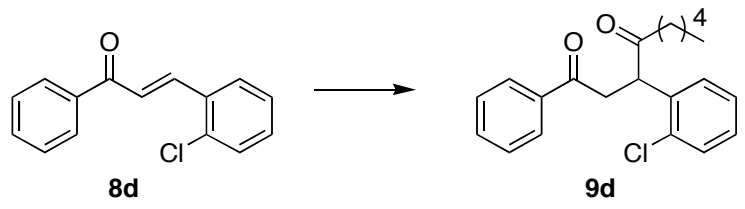

3-(2-Chlorophenyl)-1-phenylnonane-1,4-dione (361 mg, 97\% yield, 95\% purity) was obtained as a orange oil: $R_{f} 0.69\left(\mathrm{Et}_{2} \mathrm{O}\right)$; IR (thin film) $1716,1686,754 \mathrm{~cm}^{-1} ;{ }^{1} \mathrm{H} \mathrm{NMR}\left(300 \mathrm{MHz}, \mathrm{CDCl}_{3}\right) \delta 7.95(\mathrm{~d}, J=7.7 \mathrm{~Hz}$, 2H), $7.54-7.38(\mathrm{~m}, 4 \mathrm{H}), 7.23-7.19(\mathrm{~m}, 3 \mathrm{H}), 5.00(\mathrm{dd}, J=10.1 \mathrm{~Hz}, J=3.1 \mathrm{~Hz}, 1 \mathrm{H}), 3.98(\mathrm{dd}, J=17.9$ $\mathrm{Hz}, J=10.1 \mathrm{~Hz}, 1 \mathrm{H}), 3.09(\mathrm{dd}, J=17.9 \mathrm{~Hz}, J=3.1 \mathrm{~Hz}, 1 \mathrm{H}), 2.69-2.38(\mathrm{~m}, 2 \mathrm{H}), 1.67-1.42(\mathrm{~m}, 2 \mathrm{H})$, $1.34-1.21(\mathrm{~m}, 4 \mathrm{H}), 0.84(\mathrm{t}, J=6.7 \mathrm{~Hz}, 3 \mathrm{H}) ;{ }^{13} \mathrm{C}$ NMR $\left(75 \mathrm{MHz}, \mathrm{CDCl}_{3}\right) \delta$ 208.8, 197.6, 136.4, 136.0, 134.1, 133.2, 130.2, 129.3, 128.8, 128.6, 128.1, 127.5, 49.2, 42.1, 41.1, 31.2, 23.4, 22.4, 13.9; MS (CI, $\left.\mathrm{NH}_{3}\right) \mathrm{m} / z 360\left(\mathrm{M}+\mathrm{NH}_{4}\right)^{+}, 343(\mathrm{M}+\mathrm{H})^{+}$; HRMS $\left(\mathrm{CI}, \mathrm{NH}_{3}\right) \mathrm{m} / z$ calc for $\mathrm{C}_{21} \mathrm{H}_{24} \mathrm{O}_{2} \mathrm{Cl}$ : 343.1465; found: 343.1453. Anal. calc for $\mathrm{C}_{21} \mathrm{H}_{23} \mathrm{O}_{2} \mathrm{Cl}$ : C, 73.57; H, 6.76. Found: C, 73.39; H, 6.96.

\section{1,3-Di(4-chlorophenyl)nonane-1,4-dione (9e)}

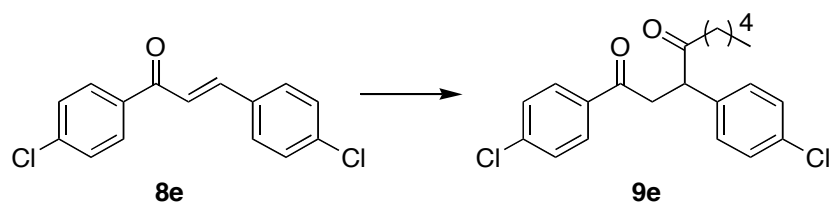

1,3-Di(4-chlorophenyl)nonane-1,4-dione (345 mg, 92\% yield, 95\% purity) was obtained as a yellow oil: $R_{f}$ 0.73 (Et $\mathrm{Et}_{2}$ ); IR (thin film) 1713, 1682, 1590, 1488, 1092, 1014, $820 \mathrm{~cm}^{-1} ;{ }^{1} \mathrm{H} \mathrm{NMR}\left(300 \mathrm{MHz}, \mathrm{CDCl}_{3}\right) \delta$ $7.87(\mathrm{~d}, J=8.3 \mathrm{~Hz}, 2 \mathrm{H}), 7.38(\mathrm{~d}, J=8.3 \mathrm{~Hz}, 2 \mathrm{H}), 7.30(\mathrm{~d}, J=8.2 \mathrm{~Hz}, 2 \mathrm{H}), 7.21(\mathrm{~d}, J=8.2 \mathrm{~Hz}, 2 \mathrm{H}), 4.38$ $(\mathrm{dd}, J=10.0 \mathrm{~Hz}, J=3.2 \mathrm{~Hz}, 1 \mathrm{H}), 3.95(\mathrm{dd}, J=18.0 \mathrm{~Hz}, J=10.0 \mathrm{~Hz}, 1 \mathrm{H}), 3.05(\mathrm{dd}, J=18.0 \mathrm{~Hz}, J=3.2$ $\mathrm{Hz}, 1 \mathrm{H}), 2.64-2.38(\mathrm{~m}, 2 \mathrm{H}), 1.60-1.18(\mathrm{~m}, 6 \mathrm{H}), 0.82(\mathrm{t}, J=6.8 \mathrm{~Hz}, 3 \mathrm{H}) ;{ }^{13} \mathrm{C} \mathrm{NMR}\left(75 \mathrm{MHz}, \mathrm{CDCl}_{3}\right) \delta$ 208.9, 196.6, 139.7, 136.5, 134.7, 133.6, 129.7, 129.5, 129.3, 128.9, 52.6, 42.2, 41.8, 31.2, 23.3, 22.4, 13.9; MS $\left(\mathrm{CI}, \mathrm{NH}_{3}\right) \mathrm{m} / z, 394\left(\mathrm{M}+\mathrm{NH}_{4}\right)^{+}, 377(\mathrm{M}+\mathrm{H})^{+}$; HRMS $\left(\mathrm{CI}, \mathrm{NH}_{3}\right) \mathrm{m} / z$ calc for $\mathrm{C}_{21} \mathrm{H}_{23} \mathrm{O}_{2} \mathrm{Cl}_{2}: 377.1075$; found: 377.1086 


\section{1,3-Di(4-methoxyphenyl)nonane-1,4-dione (9f)}

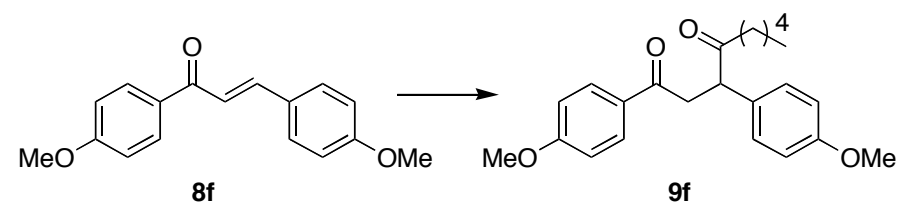

1,3-Di(4-methoxyphenyl)nonane-1,4-dione (317 mg, 86\% yield, 95\% purity) was obtained as a clear colorless oil: $R_{f} 0.10$ (1:4; Et $\mathrm{O}_{2}$ : hexanes); IR (thin film) 1713, 1678, 1600, 1253, 1169, 1032, $831 \mathrm{~cm}^{-1}$; ${ }^{1} \mathrm{H}$ NMR $\left(300 \mathrm{MHz}, \mathrm{CDCl}_{3}\right) \delta 7.93(\mathrm{~d}, J=8.8 \mathrm{~Hz}, 2 \mathrm{H}), 7.20(\mathrm{~d}, J=8.5 \mathrm{~Hz}, 2 \mathrm{H}), 6.89(\mathrm{~d}, J=6.9 \mathrm{~Hz}, 2 \mathrm{H})$, $6.87(\mathrm{~d}, J=6.9 \mathrm{~Hz}, 2 \mathrm{H}), 4.35(\mathrm{dd}, J=10.1 \mathrm{~Hz}, J=3.6 \mathrm{~Hz}, 1 \mathrm{H}), 3.95(\mathrm{dd}, J=17.8 \mathrm{~Hz}, J=10.1 \mathrm{~Hz}, 1 \mathrm{H})$, $3.82(\mathrm{~s}, 3 \mathrm{H}), 3.78(\mathrm{~s}, 3 \mathrm{H}), 3.05(\mathrm{dd}, J=17.8 \mathrm{~Hz}, J=3.6 \mathrm{~Hz}, 1 \mathrm{H}), 2.63-2.41(\mathrm{~m}, 2 \mathrm{H}), 1.61-1.46(\mathrm{~m}, 2 \mathrm{H})$, $1.29-1.14(\mathrm{~m}, 4 \mathrm{H}), 0.83(\mathrm{t}, J=6.9 \mathrm{~Hz}, 3 \mathrm{H}) ;{ }^{13} \mathrm{C} \mathrm{NMR}\left(75 \mathrm{MHz}, \mathrm{CDCl}_{3}\right) \delta 209.8,196.8,163.5,159.0$, $130.3,129.7,129.4,114.4,113.7,55.4,55.3,52.5,42.1,41.7,31.2,23.4,22.4,13.9 ; \mathrm{MS}\left(\mathrm{CI}, \mathrm{NH}_{3}\right) \mathrm{m} / z$ $369(\mathrm{M}+\mathrm{H})^{+}$; HRMS $\left(\mathrm{CI}, \mathrm{NH}_{3}\right) \mathrm{m} / z$ calc for $\mathrm{C}_{23} \mathrm{H}_{29} \mathrm{O}_{4}: 369.2066$; found: 369.2054. Anal. calc for $\mathrm{C}_{23} \mathrm{H}_{28} \mathrm{O}_{4}$ : C, 74.97; H, 7.66. Found: C, 74.98; H, 7.69.

\section{4-Phenyldecane-2,5-dione (9g)}

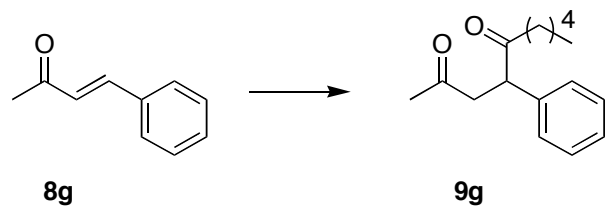

4-Phenyldecane-2,5-dione (166 mg, 95\% yield, 95\% purity) was obtained as a yellow oil: $R_{f} 0.68\left(\mathrm{Et}_{2} \mathrm{O}\right)$; IR (thin film) 1713, $701 \mathrm{~cm}^{-1} ;{ }^{1} \mathrm{H}$ NMR $\left(300 \mathrm{MHz}^{\mathrm{CDCl}}{ }_{3}\right) \delta 7.35-7.17(\mathrm{~m}, 5 \mathrm{H}), 4.22(\mathrm{dd}, J=10.3 \mathrm{~Hz}, J$ $=3.6 \mathrm{~Hz}, 1 \mathrm{H}), 3.46(\mathrm{dd}, J=17.9 \mathrm{~Hz}, J=10.3 \mathrm{~Hz}, 1 \mathrm{H}), 2.57(\mathrm{dd}, J=17.9 \mathrm{~Hz}, J=3.6 \mathrm{~Hz}, 1 \mathrm{H}), 2.49-2.38$ $(\mathrm{m}, 2 \mathrm{H}), 2.16(\mathrm{~s}, 3 \mathrm{H}), 1.64-0.90(\mathrm{~m}, 6 \mathrm{H}), 0.82(\mathrm{t}, J=6.9 \mathrm{~Hz}, 3 \mathrm{H}) ;{ }^{13} \mathrm{C} \mathrm{NMR}\left(75 \mathrm{MHz}, \mathrm{CDCl}_{3}\right) \delta 209.5$, 206.9, 138.0, 129.1, 128.2, 127.5, 53.3, 46.6, 41.5, 31.1, 30.0, 23.3, 22.3, 13.9; MS (CI, $\left.\mathrm{NH}_{3}\right) \mathrm{m} / z 264$ $\left(\mathrm{M}+\mathrm{NH}_{4}\right)^{+}, 247(\mathrm{M}+\mathrm{H})^{+}$; HRMS $\left(\mathrm{CI}, \mathrm{NH}_{3}\right) \mathrm{m} / \mathrm{z}$ calc for $\mathrm{C}_{16} \mathrm{H}_{23} \mathrm{O}_{2}: 247.1698$; found: 247.1696. Anal. calc for $\mathrm{C}_{16} \mathrm{H}_{22} \mathrm{O}_{2}$ : C, 78.01; H, 9.00. Found: C, 77.87; H, 9.08.

\section{4-Phenylpentadecane-2,5-dione (9h)}

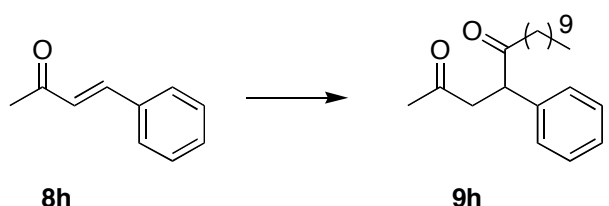

4-Phenylpentadecane-2,5-dione (334 $\mathrm{mg}, 95 \%$ yield, $90 \%$ purity) was obtained as a yellow oil: $R_{f} 0.68$ $\left(\mathrm{Et}_{2} \mathrm{O}\right)$; IR (thin film) 1715, $701 \mathrm{~cm}^{-1} ;{ }^{1} \mathrm{H}$ NMR $\left(300 \mathrm{MHz}, \mathrm{CDCl}_{3}\right) \delta 7.35-7.19(\mathrm{~m}, 5 \mathrm{H}), 4.22(\mathrm{dd}, J=$ $10.3 \mathrm{~Hz}, J=3.6 \mathrm{~Hz}, 1 \mathrm{H}), 3.47(\mathrm{dd}, J=18.0 \mathrm{~Hz}, J=10.3 \mathrm{~Hz}, 1 \mathrm{H}), 2.57$ (dd, $J=18.0 \mathrm{~Hz}, J=3.6 \mathrm{~Hz}, 1 \mathrm{H})$, $2.49-2.38(\mathrm{~m}, 2 \mathrm{H}), 2.17(\mathrm{~s}, 3 \mathrm{H}), 1.52-1.18(\mathrm{~m}, 16 \mathrm{H}), 0.88(\mathrm{t}, J=6.9 \mathrm{~Hz}, 3 \mathrm{H}) ;{ }^{13} \mathrm{C}$ NMR $(75 \mathrm{MHz}$, $\left.\mathrm{CDCl}_{3}\right) \delta$ 209.5, 206.9, 138.0, 129.1, 128.2, 127.5, 53.3, 46.6, 41.6, 31.9, 30.0, 29.5, 29.4, 29.3, 29.0, 23.6, 22.7, 14.1; MS (CI, $\left.\mathrm{NH}_{3}\right) \mathrm{m} / z 334\left(\mathrm{M}+\mathrm{NH}_{4}\right)^{+}, 317(\mathrm{M}+\mathrm{H})^{+}$; HRMS $\left(\mathrm{CI}, \mathrm{NH}_{3}\right) \mathrm{m} / z$ calc for $\mathrm{C}_{21} \mathrm{H}_{33} \mathrm{O}_{2}$ : 317.2481; found: 317.2480 . 


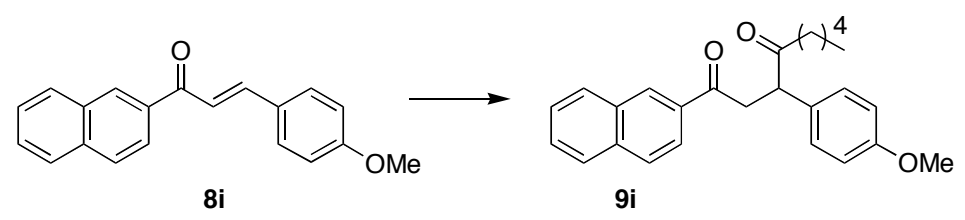

3-(4-Methoxyphenyl)-1-(2-naphthyl)nonane-1,4-dione (350 mg, 98\% yield, 85\% purity) was obtained as a yellow oil: $R_{f} 0.66\left(\mathrm{Et}_{2} \mathrm{O}\right)$; IR (thin film) 1713, 1682, 1505, 1463, 1367, 1302, 1250, $1178 \mathrm{~cm}^{-1}$; ${ }^{1} \mathrm{H}$ NMR $\left(300 \mathrm{MHz}, \mathrm{CDCl}_{3}\right) \delta 8.48(\mathrm{~s}, 1 \mathrm{H}), 8.01(\mathrm{~d}, J=8.6 \mathrm{~Hz}, 1 \mathrm{H}), 7.90(\mathrm{~d}, J=7.9 \mathrm{~Hz}, 1 \mathrm{H}), 7.83(\mathrm{~d}, J=9.3 \mathrm{~Hz}$, $2 \mathrm{H}), 7.62-7.48(\mathrm{~m}, 2 \mathrm{H}), 7.26(\mathrm{~d}, J=7.8 \mathrm{~Hz}, 2 \mathrm{H}), 6.91(\mathrm{~d}, J=7.8 \mathrm{~Hz}, 2 \mathrm{H}), 4.45(\mathrm{dd}, J=9.8 \mathrm{~Hz}, J=2.7$ $\mathrm{Hz}, 1 \mathrm{H}), 4.16(\mathrm{dd}, J=17.8 \mathrm{~Hz}, J=10.0 \mathrm{~Hz}, 1 \mathrm{H}), 3.79(\mathrm{~s}, 3 \mathrm{H}), 3.24(\mathrm{dd}, J=17.8 \mathrm{~Hz}, J=2.7 \mathrm{~Hz}, 1 \mathrm{H}), 2.68$ - $2.47(\mathrm{~m}, 2 \mathrm{H}), 1.69-1.53(\mathrm{~m}, 2 \mathrm{H}), 1.37-1.23(\mathrm{~m}, 4 \mathrm{H}), 0.91-0.84(\mathrm{~m}, 3 \mathrm{H}) ;{ }^{13} \mathrm{C} \mathrm{NMR}\left(75 \mathrm{MHz}, \mathrm{CDCl}_{3}\right)$ o $209.8,198.2,159.1,135.6,133.9,132.5,130.3,129.9,129.6,129.5,128.5,128.4,127.8,126.7,123.8$, 114.5, 55.3, 52.6, 42.5, 41.7, 31.3, 23.4, 22.5, 14.0; MS (CI, $\left.\mathrm{NH}_{3}\right) \mathrm{m} / z .406\left(\mathrm{M}+\mathrm{NH}_{4}\right)^{+}, 389(\mathrm{M}+\mathrm{H})^{+}$; HRMS (CI, $\mathrm{NH}_{3}$ ) $m / z$ calc for $\mathrm{C}_{26} \mathrm{H}_{29} \mathrm{O}_{3}: 389.2117$; found: 389.2122 .

\section{1-(1-Methyl-1H-indol-2-yl)nonane-1,4-dione $(9 \mathbf{j})$}

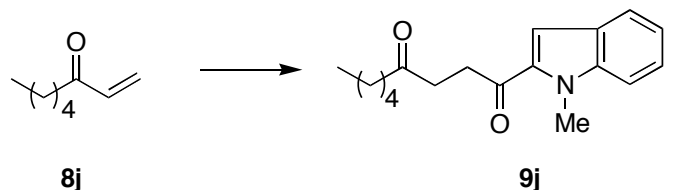

1-(1-Methyl-1H-indol-2-yl)nonane-1,4-dione (158 $\mathrm{mg}, 86 \%$ yield, $94 \%$ purity) was obtained as a white solid: $\mathrm{mp} 58.5$ - 59.5 ${ }^{\circ}\left(1: 9 ; \mathrm{Et}_{2} \mathrm{O}\right.$, hexanes). $R_{f} 0.68\left(\mathrm{Et}_{2} \mathrm{O}\right) ; \mathrm{IR}\left(\mathrm{CHCl}_{3}\right) 1712,1665,1512,1465,1396$, $751 \mathrm{~cm}^{-1} ;{ }^{1} \mathrm{H}$ NMR $\left(300 \mathrm{MHz}, \mathrm{CDCl}_{3}\right) \delta 7.72(\mathrm{~d}, J=8.1 \mathrm{~Hz}, 1 \mathrm{H}), 7.39(\mathrm{~m}, 3 \mathrm{H}), 7.19-7.16(\mathrm{~m}, 1 \mathrm{H}), 4.07$ $(\mathrm{s}, 3 \mathrm{H}), 3.34(\mathrm{t}, J=6.4 \mathrm{~Hz}, 2 \mathrm{H}), 2.87(\mathrm{t}, J=6.4 \mathrm{~Hz}, 2 \mathrm{H}), 2.54(\mathrm{t}, J=7.4 \mathrm{~Hz}, 2 \mathrm{H}), 1.67-1.62(\mathrm{~m}, 2 \mathrm{H}), 1.34$ - $1.33(\mathrm{~m}, 4 \mathrm{H}), 0.92(\mathrm{~m}, 3 \mathrm{H}) ;{ }^{13} \mathrm{C} \mathrm{NMR}\left(75 \mathrm{MHz}^{3} \mathrm{CDCl}_{3}\right) \delta$ 209.8, 192.5, 140.0, 134.5, 125.9, 123.0, 120.7, 111.4, 110.3, 43.0, 36.4, 33.4, 32.2, 31.4, 23.6, 22.5, 14.0; MS (CI, $\left.\mathrm{NH}_{3}\right) \mathrm{m} / z .286(\mathrm{M}+\mathrm{H})^{+}$; HRMS $\left(\mathrm{CI}, \mathrm{NH}_{3}\right) \mathrm{m} / \mathrm{z}$ calc for $\mathrm{C}_{18} \mathrm{H}_{24} \mathrm{NO}_{2}: 286.1807$; found: 286.1814 .

\section{2-(2-Oxo-1-phenylheptyl)-3,4-dihydronaphthalen-1(2H)-one (9k)}

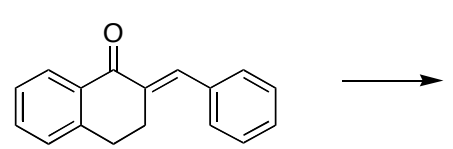

8k

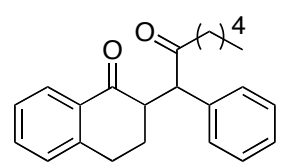

9k

2-(2-Oxo-1-phenylheptyl)-3,4-dihydronaphthalen-1(2H)-one (300 mg, 69\% yield, purity 66\%) was obtained as a yellow oil: $R_{f} 0.18$ ( $1: 9 ; \mathrm{Et}_{2} \mathrm{O}$ : hexanes); IR (thin film) 1713, 1682, 1599, 1455, 1296, 1219, 741, $702 \mathrm{~cm}^{-1}$; ${ }^{1} \mathrm{H}$ NMR $\left(300 \mathrm{MHz}, \mathrm{CDCl}_{3}\right) \delta 7.99(\mathrm{~d}, J=7.8 \mathrm{~Hz}, 1 \mathrm{H}), 7.54-7.20(\mathrm{~m}, 8 \mathrm{H}), 4.01(\mathrm{~d}, J=$ $10.0 \mathrm{~Hz}, 1 \mathrm{H}), 3.69-3.59(\mathrm{~m}, 1 \mathrm{H}), 3.10-2.80(\mathrm{~m}, 4 \mathrm{H}), 2.61-2.50(\mathrm{~m}, 2 \mathrm{H}), 1.74-1.23(\mathrm{~m}, 6 \mathrm{H}), 0.86(\mathrm{t}, J$ $=6.8 \mathrm{~Hz}, 3 \mathrm{H}) ;{ }^{13} \mathrm{C} \mathrm{NMR}\left(75 \mathrm{MHz}, \mathrm{CDCl}_{3}\right) \delta 209.5,199.5,144.0,136.4,133.4,129.0,128.9,128.7$, $128.2,127.5,127.4,126.6,58.6,51.3,42.4,31.2,29.8,27.2,23.2,22.5,13.9 ; \mathrm{MS}\left(\mathrm{CI}, \mathrm{NH}_{3}\right) \mathrm{m} / z 352$ $\left(\mathrm{M}+\mathrm{NH}_{4}\right)^{+}, 335(\mathrm{M}+\mathrm{H})^{+}$; HRMS $\left(\mathrm{CI}, \mathrm{NH}_{3}\right) \mathrm{m} / z$ calc for $\mathrm{C}_{23} \mathrm{H}_{27} \mathrm{O}_{2}: 335.2011$; found: 335.1998. 


\section{1-Cyclohexyl-2-phenylpentane-1,4-dione (91)}

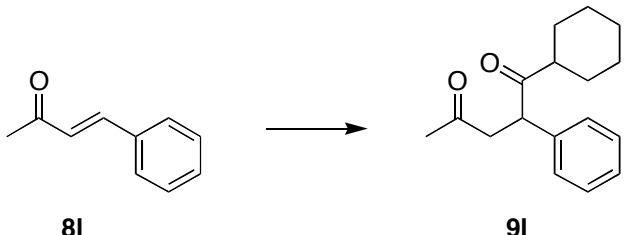

1-Cyclohexyl-2-phenylpentane-1,4-dione (229 mg, $82 \%$ yield, $82 \%$ purity) was obtained as a yellow oil: $R_{f}$ $0.70\left(\mathrm{Et}_{2} \mathrm{O}\right)$; IR (thin film) 1708, 1450, $700 \mathrm{~cm}^{-1}$; ${ }^{\mathrm{H}} \mathrm{NMR}\left(300 \mathrm{MHz}, \mathrm{CDCl}_{3}\right) \delta 7.43-7.17$ (m, 5H), 4.38 $(\mathrm{dd}, J=10.3 \mathrm{~Hz}, J=3.6 \mathrm{~Hz}, 1 \mathrm{H}), 3.44(\mathrm{dd}, J=17.9 \mathrm{~Hz}, J=10.3 \mathrm{~Hz}, 1 \mathrm{H}), 2.54(\mathrm{dd}, J=17.9 \mathrm{~Hz}, J=3.6$ $\mathrm{Hz}, 1 \mathrm{H}), 2.48-2.30(\mathrm{~m}, 1 \mathrm{H}), 2.16(\mathrm{~s}, 3 \mathrm{H}), 2.12-2.05(\mathrm{~m}, 4 \mathrm{H}), 1.87-1.06(\mathrm{~m}, 6 \mathrm{H}) ;{ }^{13} \mathrm{C}$ NMR $(75 \mathrm{MHz}$, $\left.\mathrm{CDCl}_{3}\right) \delta$ 212.4, 206.9, 138.0, 129.0, 128.3, 127.4, 51.9, 49.7, 47.0, 30.1, 29.4, 28.3, 25.9, 25.8, 25.3; MS $\left(\mathrm{CI}, \mathrm{NH}_{3}\right) \mathrm{m} / z 276\left(\mathrm{M}+\mathrm{NH}_{4}\right)^{+}, 259(\mathrm{M}+\mathrm{H})^{+}$; HRMS $\left(\mathrm{CI}, \mathrm{NH}_{3}\right) \mathrm{m} / z$ calc for $\mathrm{C}_{17} \mathrm{H}_{26} \mathrm{NO}_{2}:$ 276.1964; found: 276.1970 .

\section{1,2,4-Triphenylbutane-1,4-dione $(9 \mathrm{~m})^{2}$}
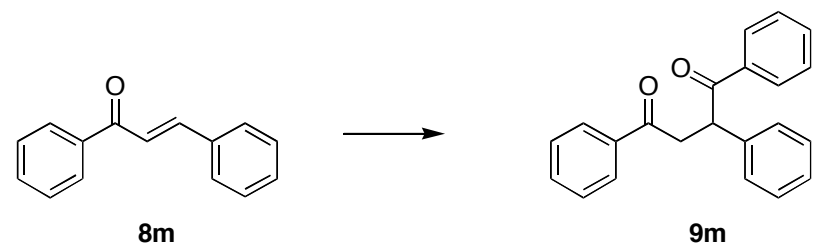

1,2,4-Triphenylbutane-1,4-dione $\left(88.1 \mathrm{mg}, 68 \%\right.$ yield, $73 \%$ purity) was obtained as a yellow oil: $R_{f} 0.62$ ( $\mathrm{Et}_{2} \mathrm{O}$ ); IR (thin film) 1678, 1597, 1448, 1230, 754, $698 \mathrm{~cm}^{-1} ;{ }^{1} \mathrm{H}$ NMR (300 MHz, $\left.\mathrm{CDCl}_{3}\right) \delta 8.08-8.00$ $(\mathrm{m}, 4 \mathrm{H}), 7.58-7.56(\mathrm{~m}, 1 \mathrm{H}), 7.52-7.23(\mathrm{~m}, 9 \mathrm{H}), 5.36(\mathrm{dd}, J=10.1 \mathrm{~Hz}, J=3.5 \mathrm{~Hz}, 1 \mathrm{H}), 4.25(\mathrm{dd}, J=$ $18.1 \mathrm{~Hz}, J=10.1 \mathrm{~Hz}, 1 \mathrm{H}), 3.34(\mathrm{dd}, J=18.1 \mathrm{~Hz}, J=3.5 \mathrm{~Hz}, 1 \mathrm{H}) ;{ }^{13} \mathrm{C}$ NMR $\left(75 \mathrm{MHz}, \mathrm{CDCl}_{3}\right) \delta 199.0$, 198.1, 138.7, 136.5, 133.3, 132.9, 129.2, 129.0, 128.7, 128.6, 128.5, 128.3, 128.2, 127.4, 48.7, 43.9; MS $\left(\mathrm{CI}, \mathrm{NH}_{3}\right) \mathrm{m} / z 332\left(\mathrm{M}+\mathrm{NH}_{4}\right)^{+}, 315(\mathrm{M}+\mathrm{H})^{+}$; HRMS $\left(\mathrm{CI}, \mathrm{NH}_{3}\right) \mathrm{m} / z$ calc for $\mathrm{C}_{22} \mathrm{H}_{19} \mathrm{O}_{2}: 315.1385$; found: 315.1390 .

(2) (a) Henrick, C. A; Ridley, D. D; Ritchie, E; Taylor, W. C Aust. J. Chem., 1970, 23, 329-339. (b) Zdrojewski, T; Jonczyk, A. Synthesis 1990, 224233. (c) Mattson, A. E.; Bharadwaj, A. R.; Scheidt, K. A. J. Am. Chem. Soc. 2004, 126, 2314-2315. 


\section{1-(4-Chlorophenyl)-3-phenyltetradecane-1,4-dione (9n)}

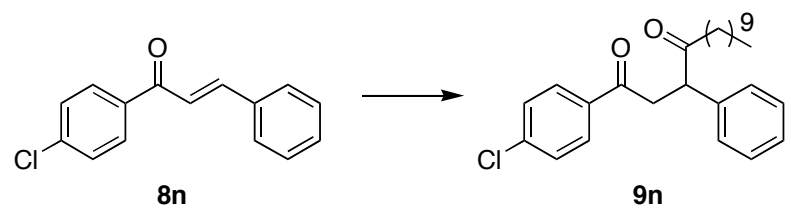

1-(4-Chlorophenyl)-3-phenyltetradecane-1,4-dione (339 $\mathrm{mg}, 97 \%$ yield, 95\% purity) was obtained as a colorless oil: $R_{f} 0.17$ (1: 9; $\mathrm{Et}_{2} \mathrm{O}$ : hexanes); IR (thin film) 1713, 1682, 1590, 1401, 1092, $700 \mathrm{~cm}^{-1} ;{ }^{1} \mathrm{H}$ NMR $\left(300 \mathrm{MHz} \mathrm{CDCl}_{3}\right) \delta 7.91(\mathrm{~d}, J=8.4 \mathrm{~Hz}, 2 \mathrm{H}), 7.43(\mathrm{~d}, J=8.4 \mathrm{~Hz}, 2 \mathrm{H}), 7.40-7.27(\mathrm{~m}, 5 \mathrm{H}), 4.43$ $(\mathrm{dd}, J=10.1 \mathrm{~Hz}, J=3.4 \mathrm{~Hz}, 1 \mathrm{H}), 4.01(\mathrm{dd}, J=17.9 \mathrm{~Hz}, J=10.1 \mathrm{~Hz}, 1 \mathrm{H}), 3.08(\mathrm{dd}, J=17.9 \mathrm{~Hz}, J=3.4$ $\mathrm{Hz}, 1 \mathrm{H}), 2.64-2.42(\mathrm{~m}, 2 \mathrm{H}), 1.57-1.53(\mathrm{~m}, 2 \mathrm{H}), 1.21(\mathrm{br} \mathrm{s}, 14 \mathrm{H}), 0.89(\mathrm{t}, J=6.4 \mathrm{~Hz}, 3 \mathrm{H}) ;{ }^{13} \mathrm{C}$ NMR $(75$ $\left.\mathrm{MHz}, \mathrm{CDCl}_{3}\right) \delta$ 209.4, 197.0, 139.6, 138.1, 134.9, 129.5, 129.1, 128.9, 128.3, 127.6, 53.4, 42.3, 41.8, 31.9, 29.6, 29.5, 29.3, 29.0, 23.7, 22.7, 14.1; MS (CI, $\left.\mathrm{NH}_{3}\right) \mathrm{m} / z, 430\left(\mathrm{M}+\mathrm{NH}_{4}\right)^{+}, 413(\mathrm{M}+\mathrm{H})^{+}$; HRMS (CI, $\mathrm{NH}_{3}$ ) $m / z$ calc for $\mathrm{C}_{26} \mathrm{H}_{34} \mathrm{O}_{2} \mathrm{Cl}$ : 413.2247; found: 413.2264: Anal. calc for $\mathrm{C}_{26} \mathrm{H}_{33} \mathrm{O}_{2} \mathrm{Cl}$ : C, 75.61; $\mathrm{H}, 8.05$. Found: C, 75.69; H, 7.85.

\section{3-(4-Chlorophenyl)-1-phenyltetradecane-1,4-dione (9o)}

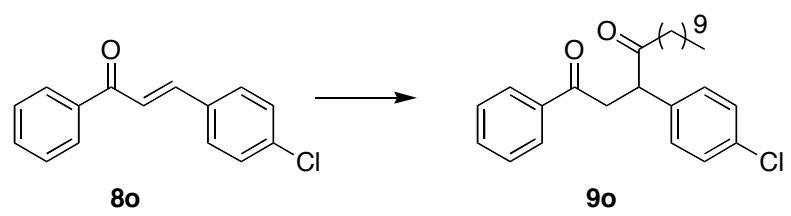

3-(4-Chlorophenyl)-1-phenyltetradecane-1,4-dione (342 mg, 99\% yield, 95\% purity) was obtained as a yellow oil: $R_{f} 0.19$ (1: 9; Et $\mathrm{O}_{2}$ : hexanes); IR (thin film) 1715, 1682, 1489, 1449, 1092, 1015, $689 \mathrm{~cm}^{-1} ;{ }^{1} \mathrm{H}$ NMR $\left(300 \mathrm{MHz}, \mathrm{CDCl}_{3}\right) \delta 7.96(\mathrm{~d}, J=7.9 \mathrm{~Hz}, 2 \mathrm{H}), 7.58-7.54(\mathrm{~m}, 1 \mathrm{H}), 7.47-7.42(\mathrm{~m}, 2 \mathrm{H}), 7.33(\mathrm{~d}, J=$ $8.3 \mathrm{~Hz}, 2 \mathrm{H}), 7.24(\mathrm{~d}, J=8.3 \mathrm{~Hz}, 2 \mathrm{H}), 4.42(\mathrm{dd}, J=9.9 \mathrm{~Hz}, J=3.6 \mathrm{~Hz}, 1 \mathrm{H}), 4.01(\mathrm{dd}, J=18.0 \mathrm{~Hz}, J=9.9$ $\mathrm{Hz}, 1 \mathrm{H}), 3.13(\mathrm{dd}, J=18.0 \mathrm{~Hz}, J=3.6 \mathrm{~Hz}, 1 \mathrm{H}), 2.66-2.42(\mathrm{~m}, 2 \mathrm{H}), 1.57-1.53(\mathrm{~m}, 2 \mathrm{H}), 1.25-1.22(\mathrm{~m}$, $14 \mathrm{H}), 0.89(\mathrm{t}, J=6.4 \mathrm{~Hz}, 3 \mathrm{H}) ;{ }^{13} \mathrm{C}$ NMR $\left(75 \mathrm{MHz}, \mathrm{CDCl}_{3}\right) \delta 209.1,197.9,136.7,136.4,133.5,133.3$, 129.7, 129.3, 128.6, 128.1 , 52.6, 42.3 42.0, 31.9, 29.6, 29.5, 29.3, 29.0, 23.6, 22.7, 14.2; MS (CI, NH $)^{2} \mathrm{~m} / z$ $430\left(\mathrm{M}+\mathrm{NH}_{4}\right)^{+}, 413(\mathrm{M}+\mathrm{H})^{+}$; HRMS $\left(\mathrm{CI}, \mathrm{NH}_{3}\right) \mathrm{m} / z$ calc for $\mathrm{C}_{26} \mathrm{H}_{34} \mathrm{O}_{2} \mathrm{Cl}$ : 413.2247; found: 413.2263: Anal. calc for $\mathrm{C}_{26} \mathrm{H}_{33} \mathrm{O}_{2} \mathrm{Cl}$ : C, 75.61; H, 8.05. Found: C, 75.74; H, 8.10. 


\section{1-(4-Methoxyphenyl)-3-phenyltetradecane-1,4-dione (9p)}

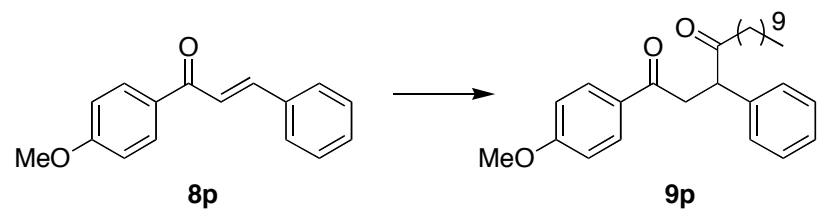

1-(4-Methoxyphenyl)-3-phenyltetradecane-1,4-dione (316 mg, 94\% yield, 95\% purity) was obtained as a yellow oil: $R_{f} 0.57\left(\mathrm{Et}_{2} \mathrm{O}\right)$; IR (thin film) 1714, 1676, 1601, 1576, 1509, 1258, 1168, $701 \mathrm{~cm}^{-1}$; ${ }^{1} \mathrm{H}$ NMR $\left(300 \mathrm{MHz}, \mathrm{CDCl}_{3}\right) \delta 7.95(\mathrm{~d}, J=8.8 \mathrm{~Hz}, 2 \mathrm{H}), 7.38-7.29(\mathrm{~m}, 5 \mathrm{H}), 6.92(\mathrm{~d}, J=8.8 \mathrm{~Hz}, 2 \mathrm{H}), 4.43(\mathrm{dd}, J=$ $10.1 \mathrm{~Hz}, J=3.3 \mathrm{~Hz}, 1 \mathrm{H}), 4.00(\mathrm{dd}, J=17.8 \mathrm{~Hz}, J=10.1 \mathrm{~Hz}, 1 \mathrm{H}), 3.86(\mathrm{~s}, 3 \mathrm{H}), 3.09(\mathrm{dd}, J=17.8 \mathrm{~Hz}, J=$ $3.3 \mathrm{~Hz}, 1 \mathrm{H}), 2.66-2.43(\mathrm{~m}, 2 \mathrm{H}), 1.60-1.53(\mathrm{~m}, 2 \mathrm{H}), 1.21(\mathrm{~m}, 14 \mathrm{H}), 0.89(\mathrm{~m}, 3 \mathrm{H}) ;{ }^{13} \mathrm{C}$ NMR $(75 \mathrm{MHz}$, $\left.\mathrm{CDCl}_{3}\right) \delta$ 209.6, 196.7, 163.6, 138.4, 130.4, 129.7, 129.1, 128.4, 127.4, 113.7, 55.4, 53.4, 42.1, 41.9, 31.9, 29.6, 29.5, 29.3, 29.0, 23.7, 22.7, 14.1; $\mathrm{MS}\left(\mathrm{CI}, \mathrm{NH}_{3}\right) \mathrm{m} / z$ $409(\mathrm{M}+\mathrm{H})^{+}$; HRMS $\left(\mathrm{CI}, \mathrm{NH}_{3}\right) \mathrm{m} / z$ calc for $\mathrm{C}_{27} \mathrm{H}_{37} \mathrm{O}_{3}: 409.2743$; found: 409.2741 .

\section{3-(4-Methoxyphenyl)-1-phenyltetradecane-1,4-dione (9q)}
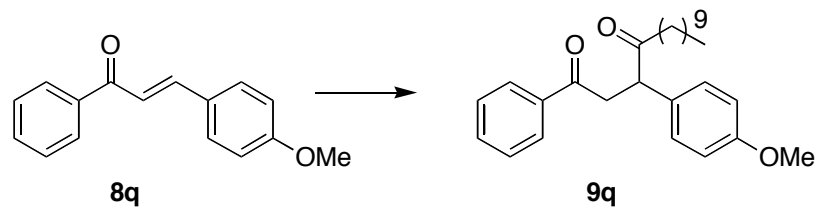

3-(4-Methoxyphenyl)-1-phenyltetradecane-1,4-dione (295 $\mathrm{mg}, 87 \%$ yield, 95\% purity) was obtained as a orange oil: $R_{f} 0.14$ (1:9; $\mathrm{Et}_{2} \mathrm{O}:$ hexanes); IR (thin film) $1714,1685,1511,1254 \mathrm{~cm}^{-1} ;{ }^{1} \mathrm{H}$ NMR (300 $\left.\mathrm{MHz}, \mathrm{CDCl}_{3}\right) \delta 7.97(\mathrm{~d}, J=7.2 \mathrm{~Hz}, 2 \mathrm{H}), 7.57-7.42(\mathrm{~m}, 3 \mathrm{H}), 7.21(\mathrm{~d}, J=8.6 \mathrm{~Hz}, 2 \mathrm{H}), 6.89(\mathrm{~d}, J=8.6$ $\mathrm{Hz}, 2 \mathrm{H}), 4.38(\mathrm{dd}, J=10.0 \mathrm{~Hz}, J=3.5 \mathrm{~Hz}, 1 \mathrm{H}), 4.01(\mathrm{dd}, J=18.0 \mathrm{~Hz}, J=10.0 \mathrm{~Hz}, 1 \mathrm{H}), 3.80(\mathrm{~s}, 3 \mathrm{H}), 3.11$ $(\mathrm{dd}, J=18.0 \mathrm{~Hz}, J=3.5 \mathrm{~Hz}, 1 \mathrm{H}), 2.64-2.43(\mathrm{~m}, 2 \mathrm{H}), 1.60-1.51(\mathrm{~m}, 2 \mathrm{H}), 1.28-1.22(\mathrm{~m}, 14 \mathrm{H}), 0.91-$ $0.87(\mathrm{~m}, 3 \mathrm{H}) ;{ }^{13} \mathrm{C}$ NMR $\left(75 \mathrm{MHz}, \mathrm{CDCl}_{3}\right) \delta$ 209.7, 198.4, 159.0, 136.6, 133.1, 130.2, 129.4, 128.5, 128.1, $114.5,55.3,55.2,52.5,42.4,41.7,31.9,29.6,29.5,29.3,29.1,23.7,22.7,14.1 ; \mathrm{MS}\left(\mathrm{CI}, \mathrm{NH}_{3}\right) \mathrm{m} / z .426$ $\left(\mathrm{M}+\mathrm{NH}_{4}\right)^{+}, 409(\mathrm{M}+\mathrm{H})^{+}$; HRMS $\left(\mathrm{CI}, \mathrm{NH}_{3}\right) \mathrm{m} / z$ calc for $\mathrm{C}_{27} \mathrm{H}_{37} \mathrm{O}_{3}: 409.2743$; found: 409.2729: Anal. calc for $\mathrm{C}_{27} \mathrm{H}_{36} \mathrm{O}_{3}$ : C, 79.37; $\mathrm{H}, 8.88$. Found: C, 79.45; H, 9.00. 


\section{Additional Spectra}

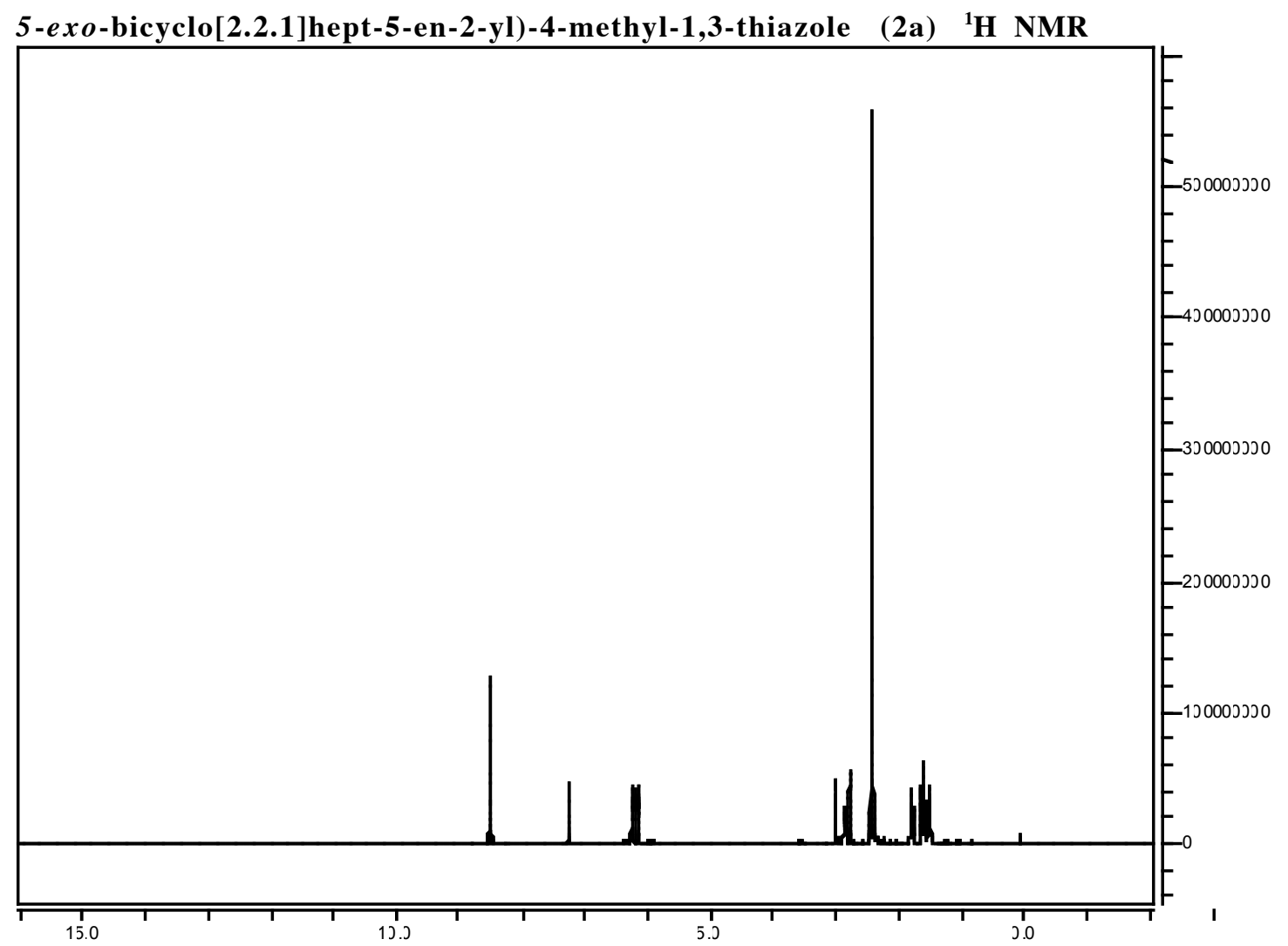

$$
\text { эрm;:1) }
$$

5-exo-bicyclo[2.2.1]hept-5-en-2-yl)-4-methyl-1,3-thiazole $\quad(2 a){ }^{13} \mathrm{C}$ NMR

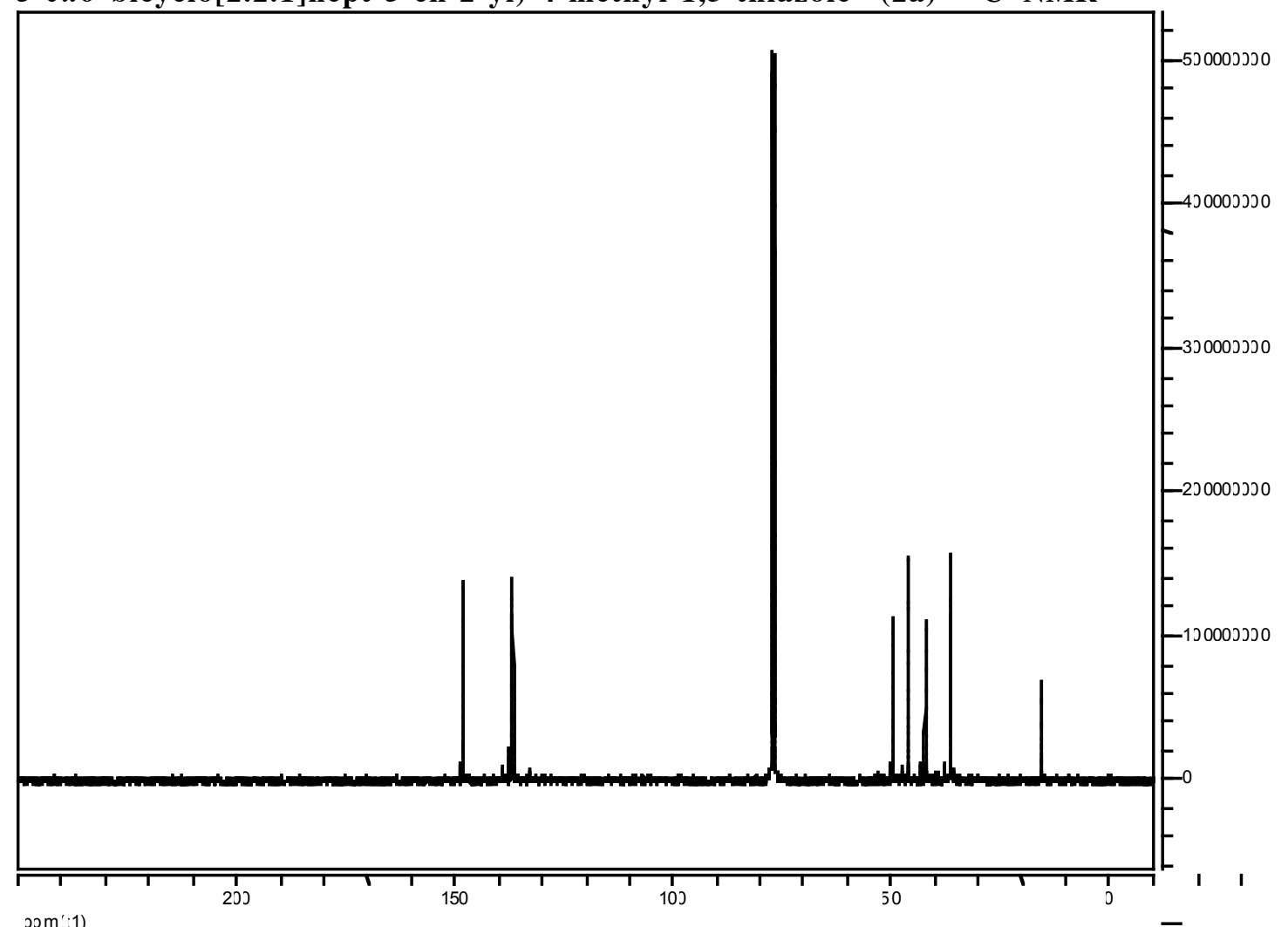

วрm(:1) 


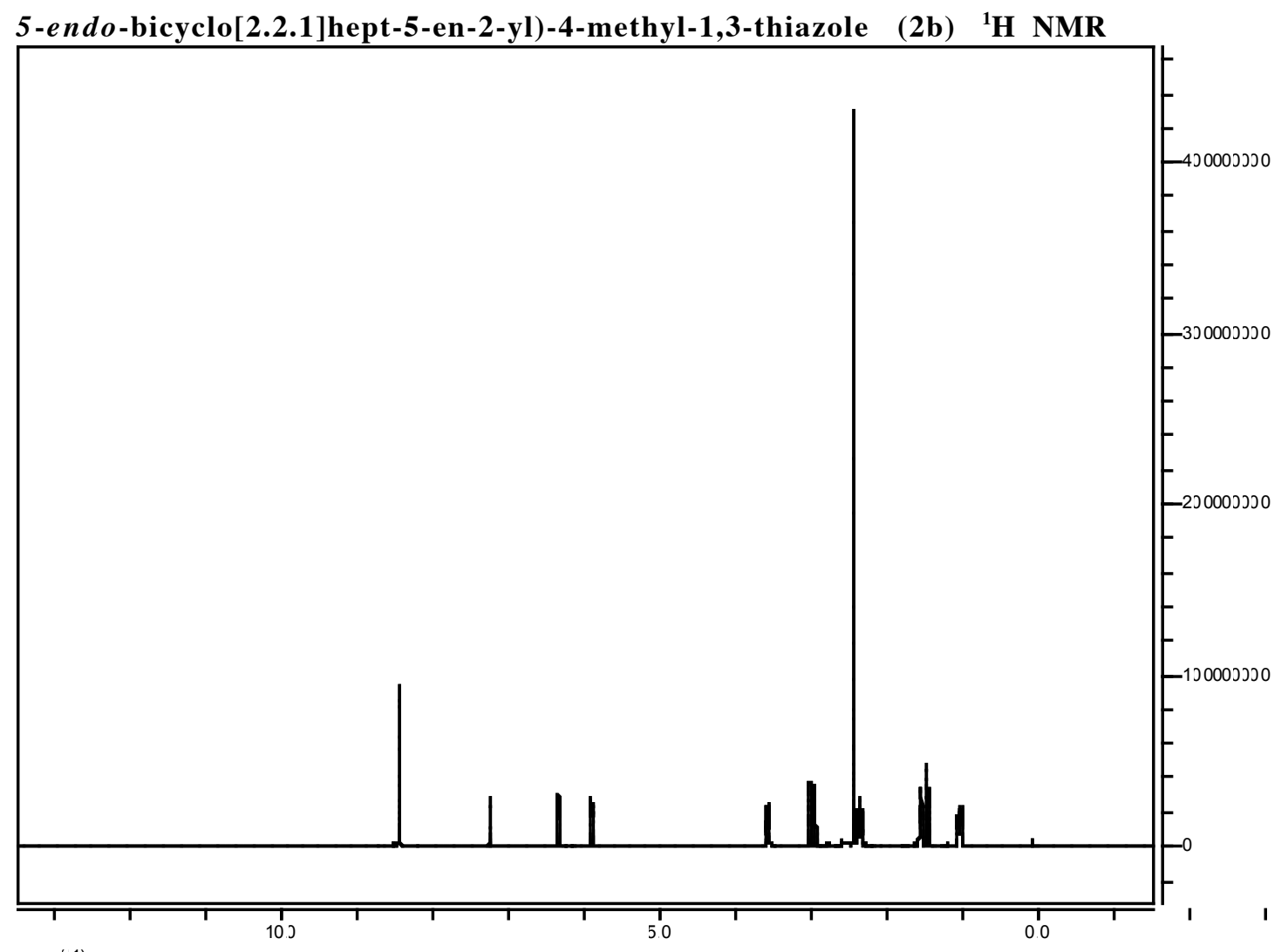

วom':1)

5-endo-bicyclo[2.2.1]hept-5-en-2-yl)-4-methyl-1,3-thiazole $\quad(2 \mathrm{~b}){ }^{13} \mathrm{C}$ NMR

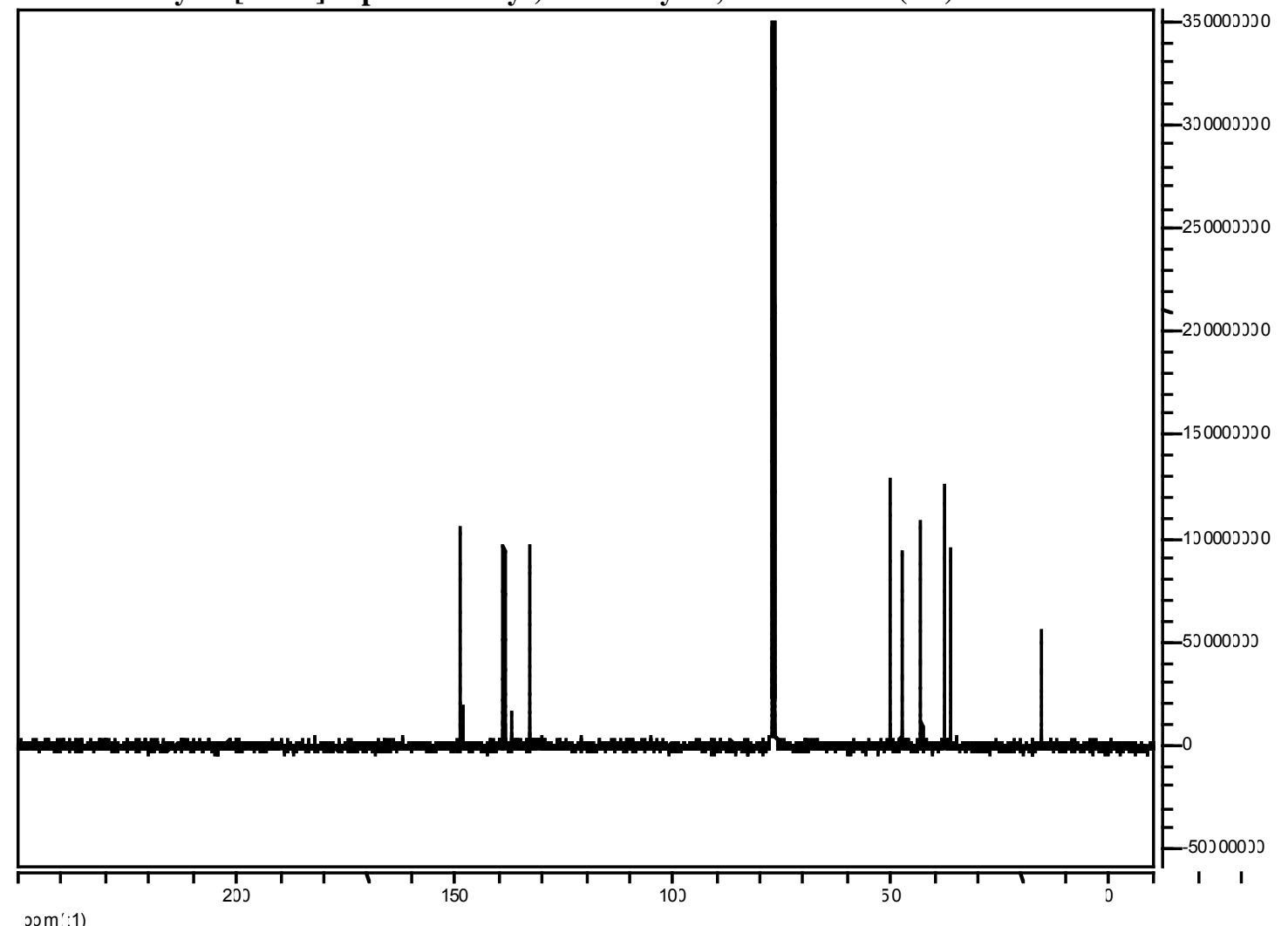




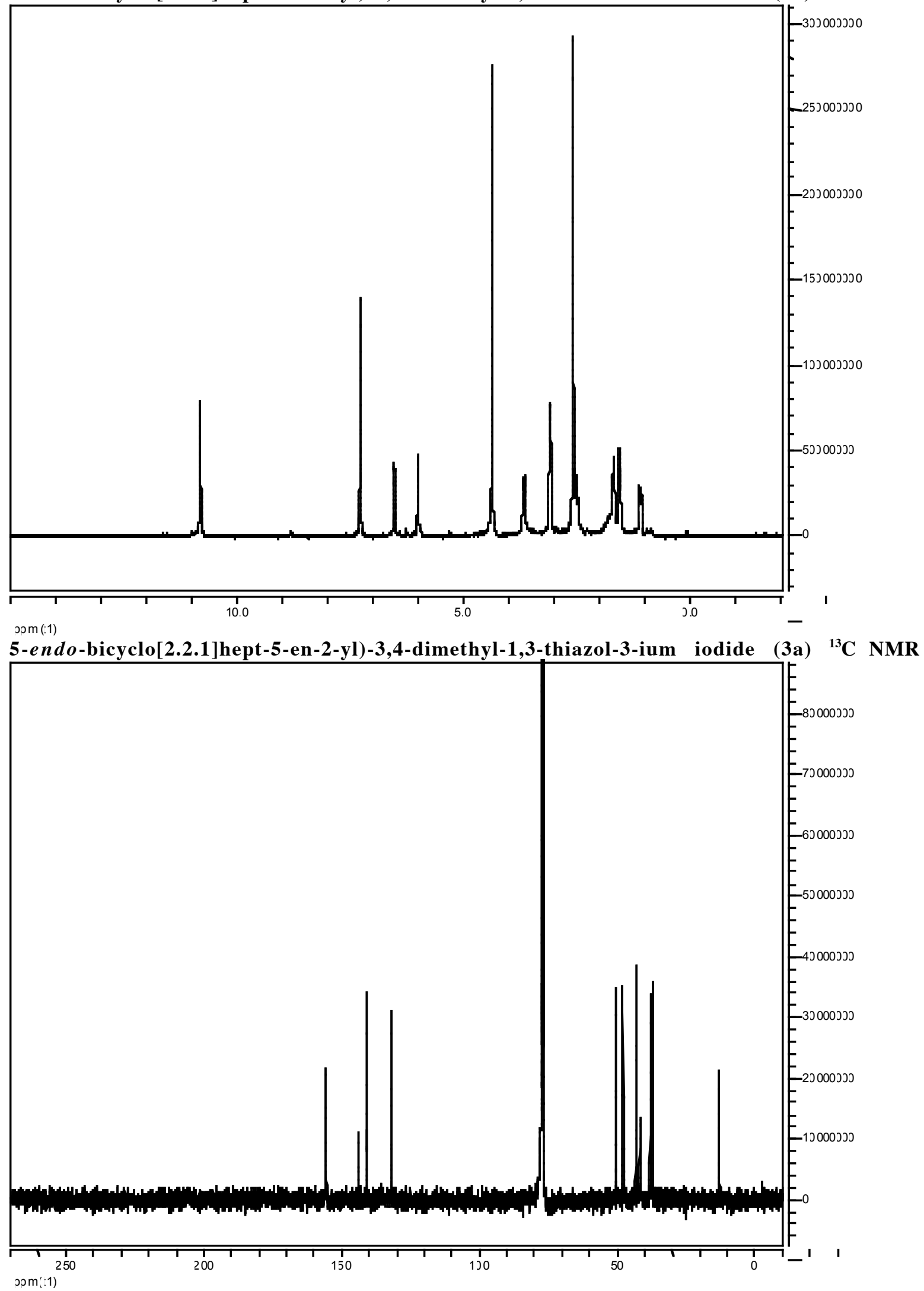




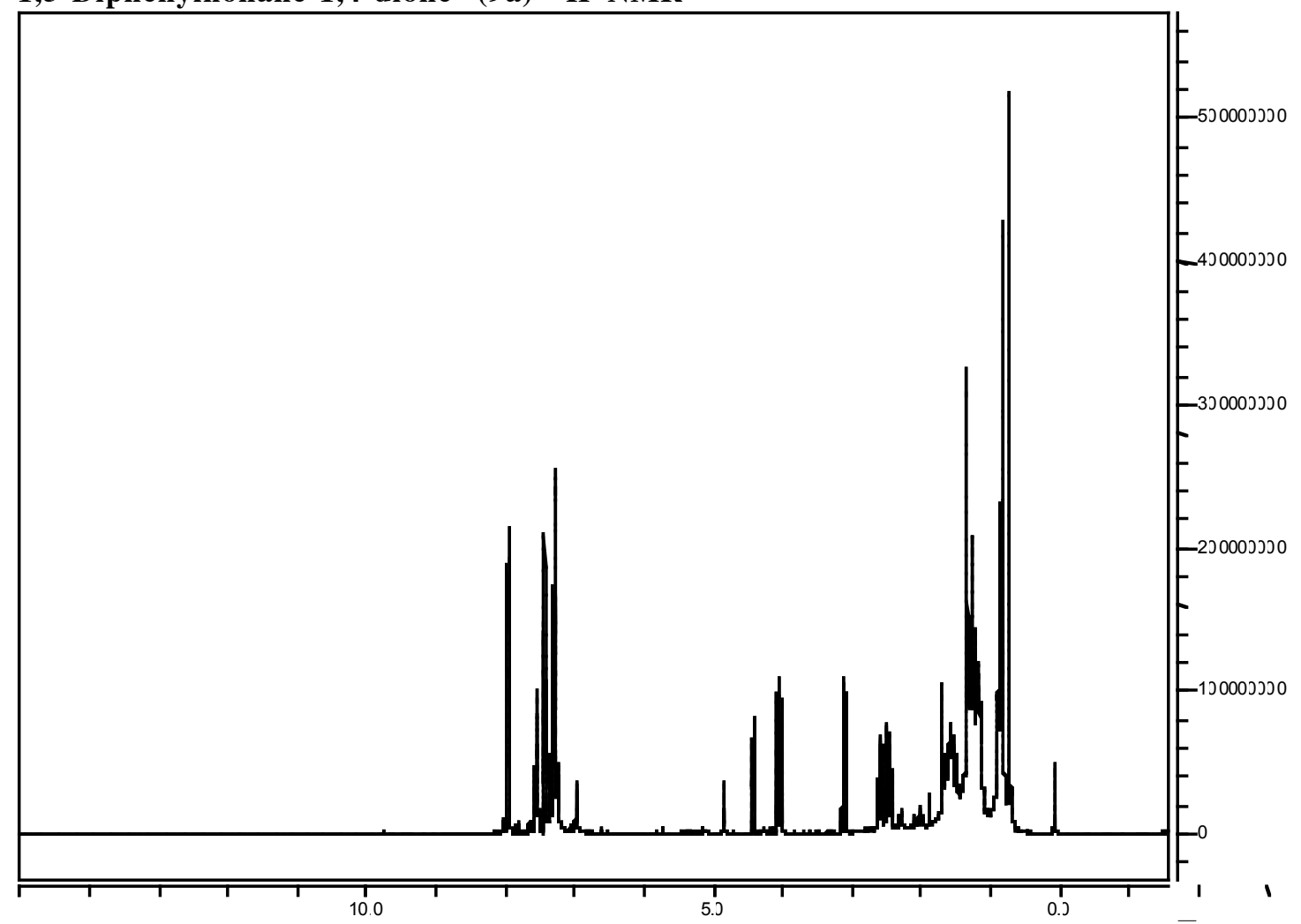

1,3-Diphenylnonane-1,4-dione $(9 a){ }^{13} \mathrm{C}$ NMR

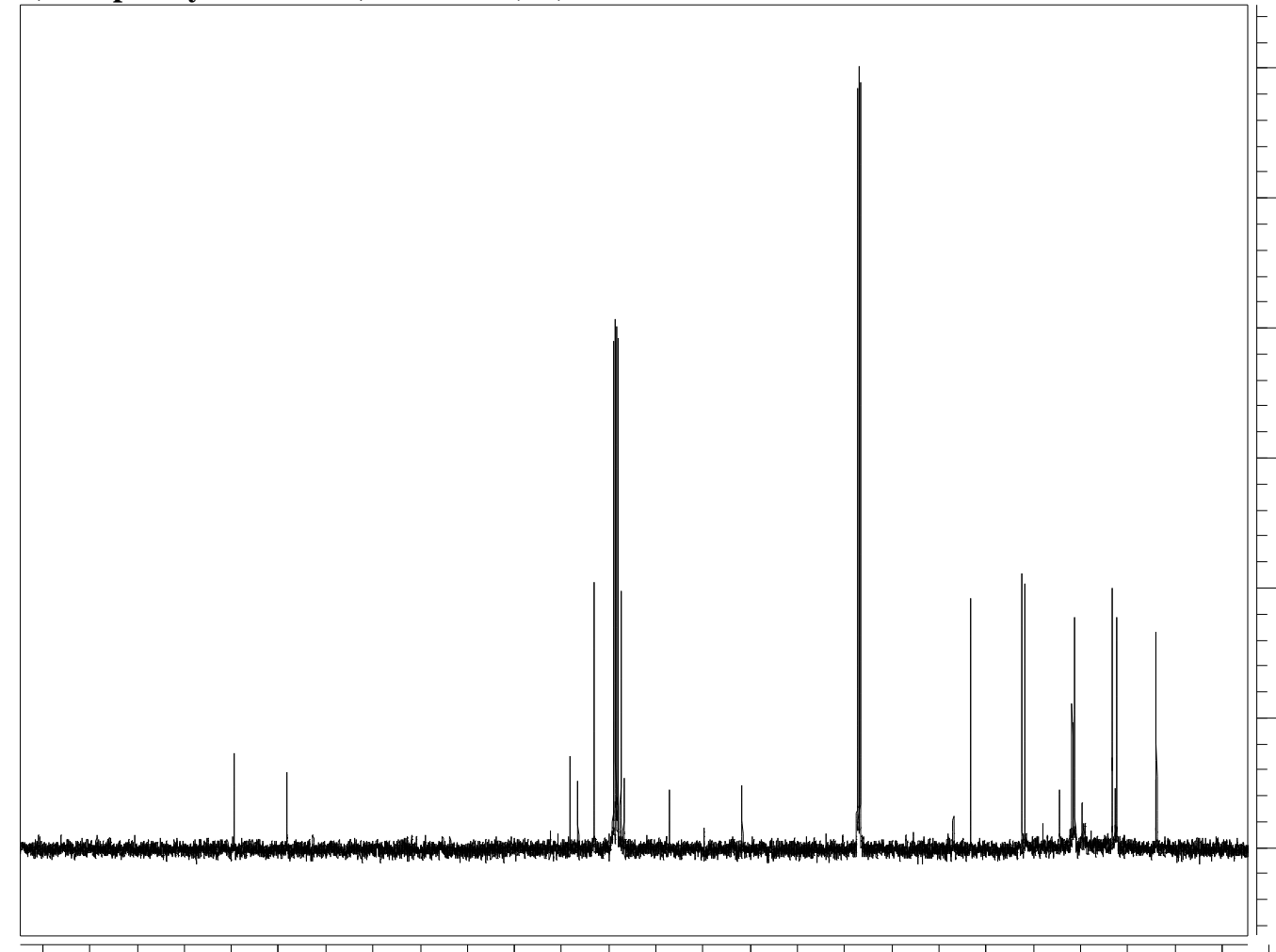




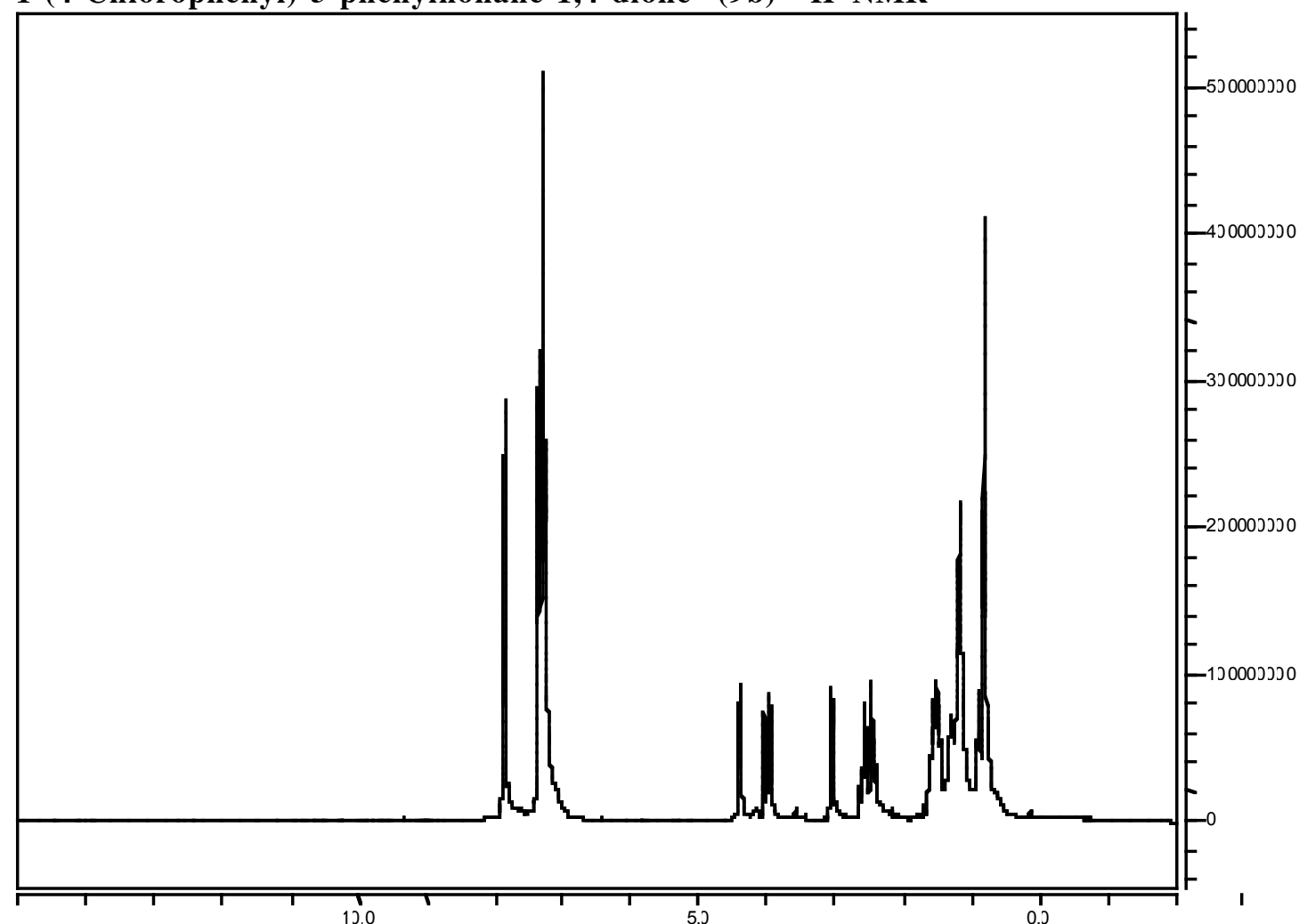

эот':1)

1-(4-Chlorophenyl)-3-phenylnonane-1,4-dione $(9 \mathrm{~b}){ }^{13} \mathrm{C}$ NMR

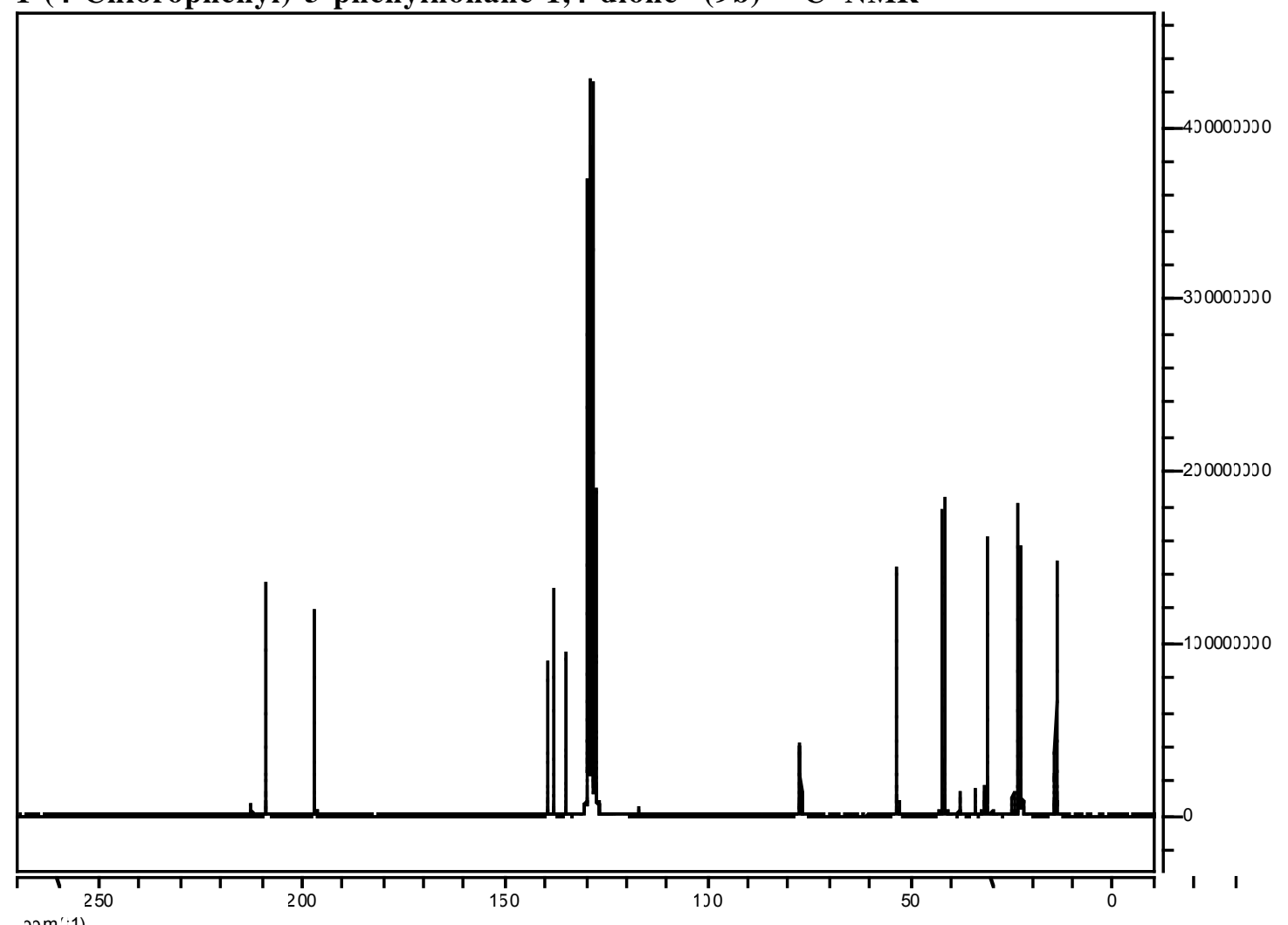

วоm $\left.\mathrm{m}^{\prime}: 1\right)$ 


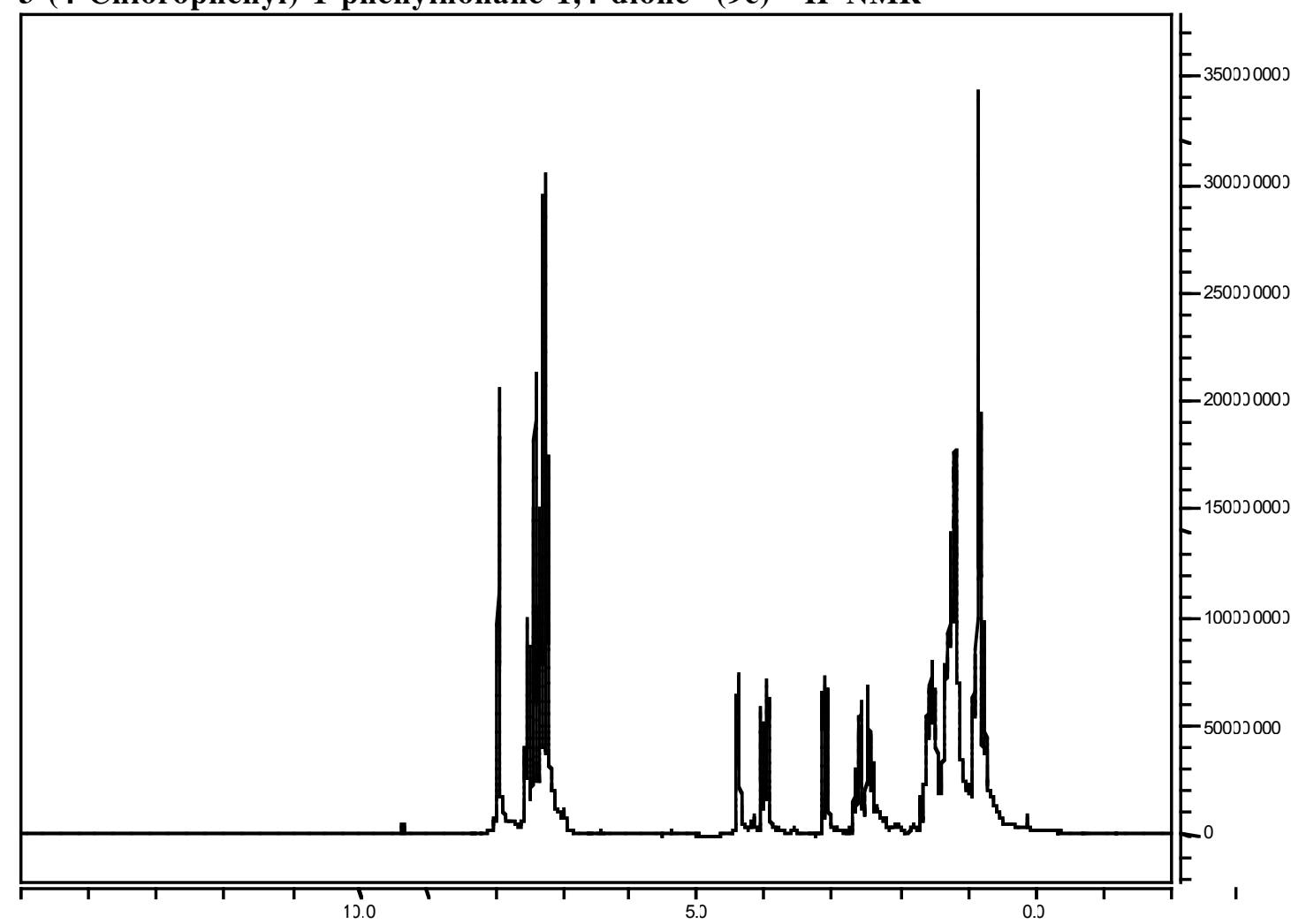

วрm::1)

3-(4-Chlorophenyl)-1-phenylnonane-1,4-dione $(9 \mathrm{c}){ }^{13} \mathrm{C}$ NMR

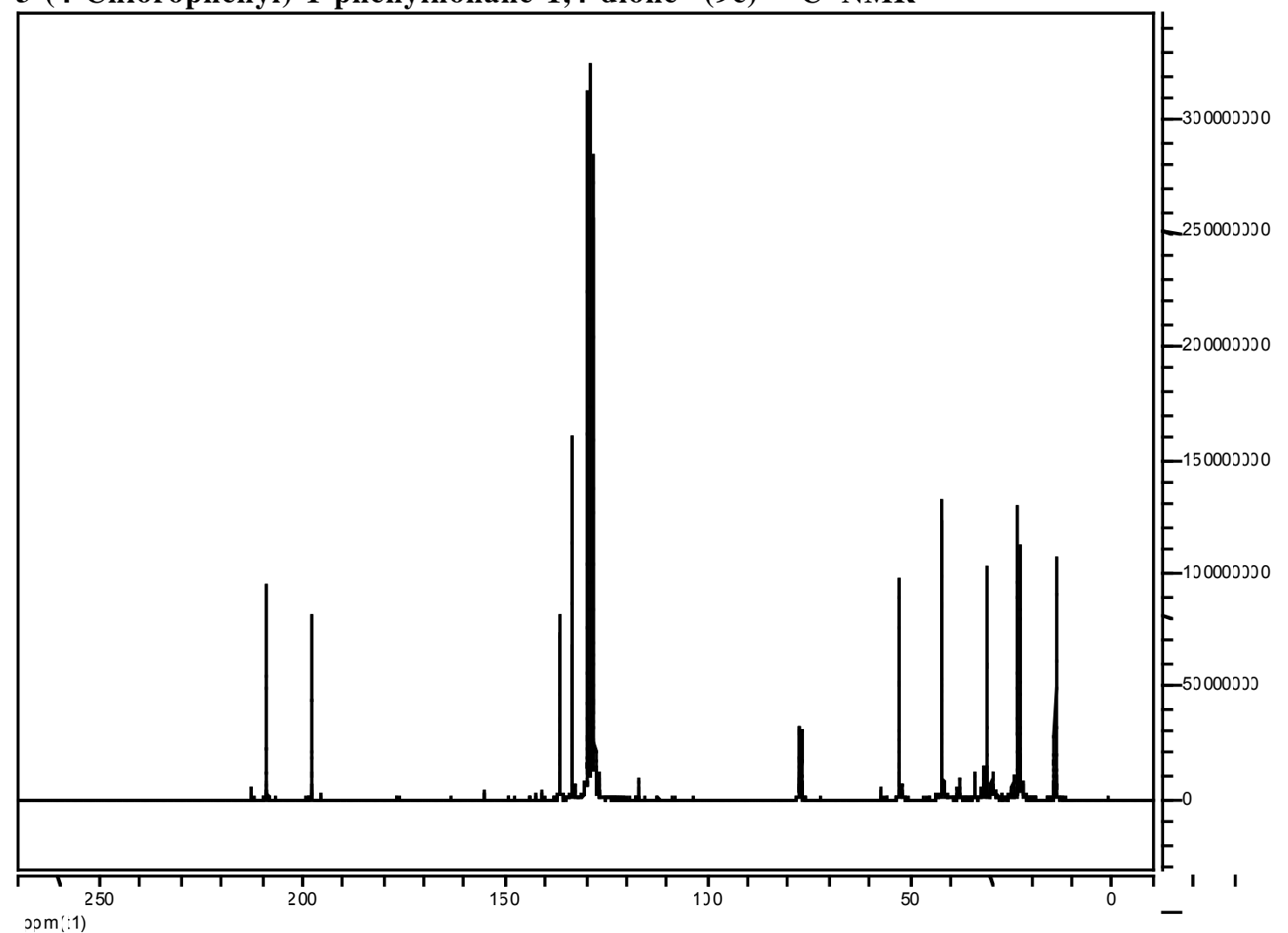

วоm':1) 


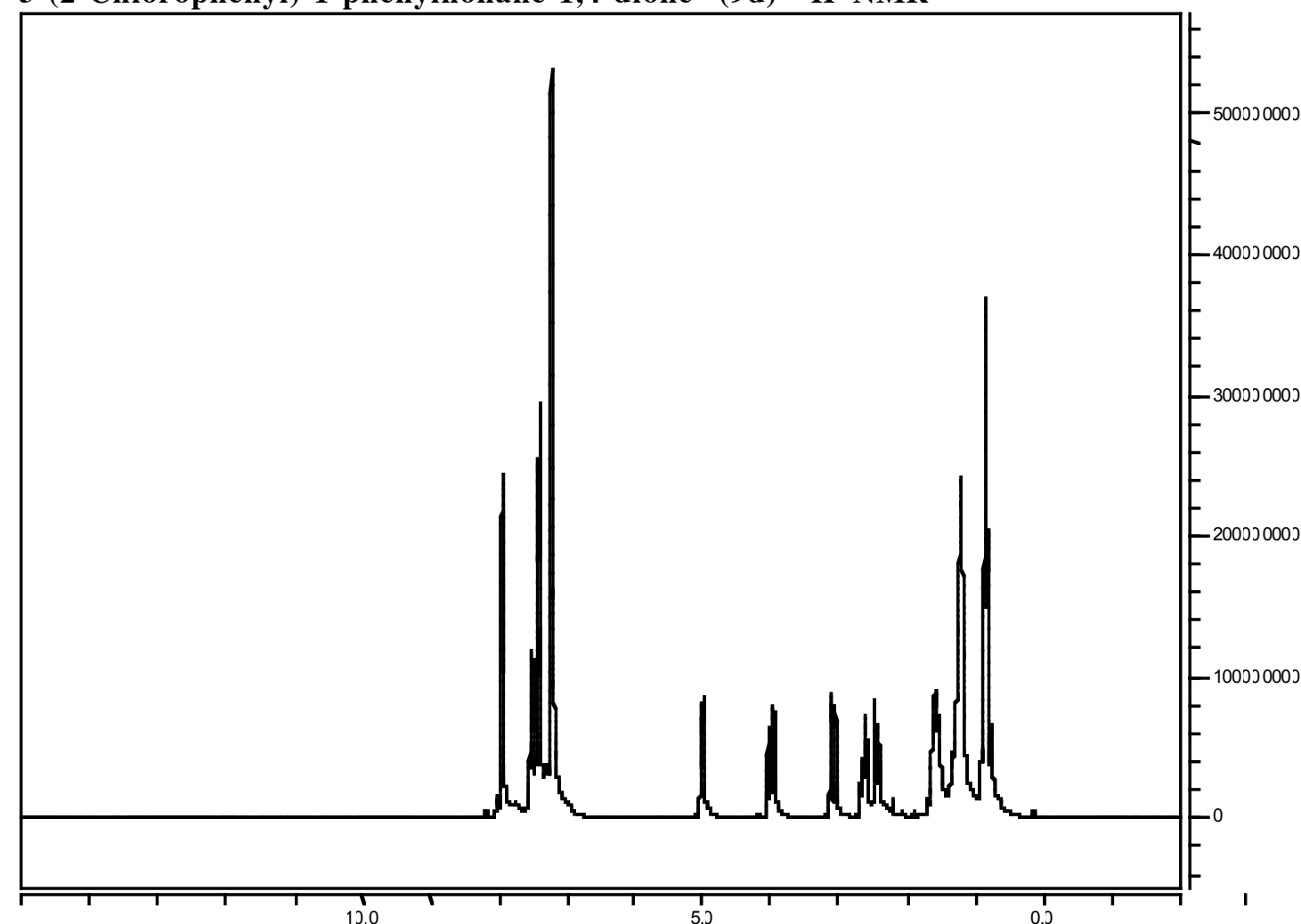

эрт ::1)

3-(2-Chlorophenyl)-1-phenylnonane-1,4-dione $\quad$ (9d) ${ }^{13} \mathrm{C}$ NMR

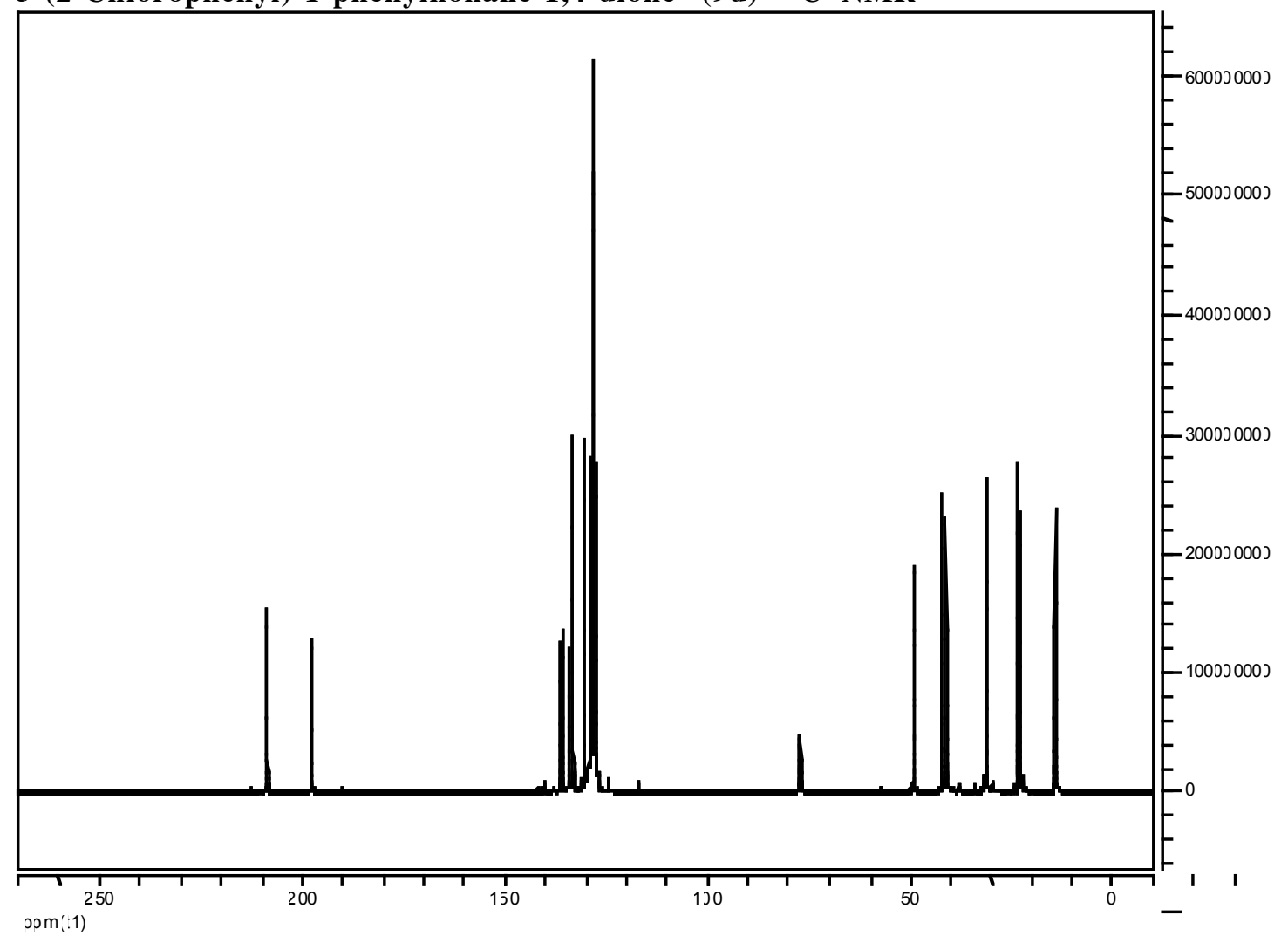

эpm: (1) 


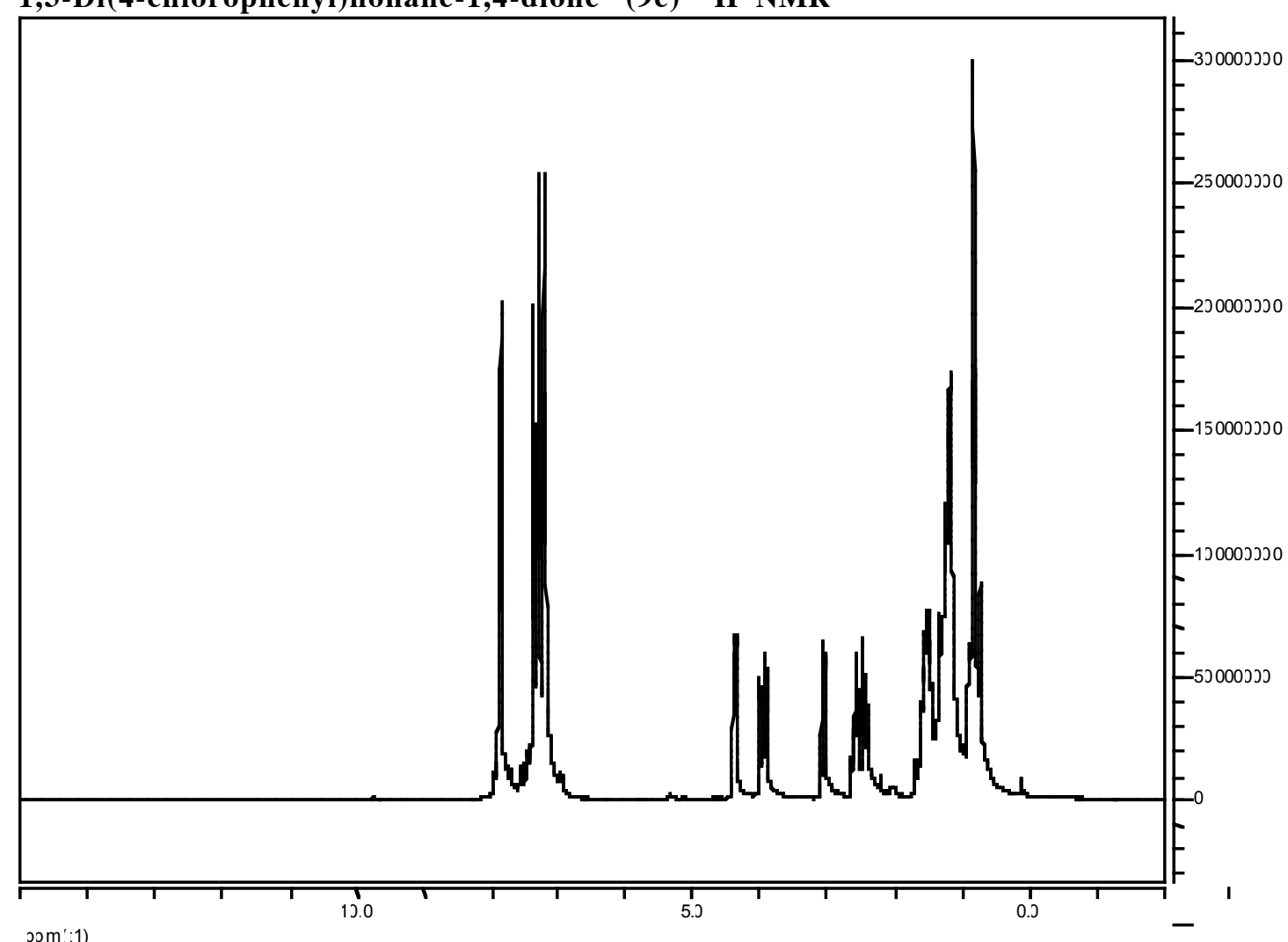

эрт ::1)

1,3-Di(4-chlorophenyl)nonane-1,4-dione (9e) ${ }^{13} \mathrm{C}$ NMR

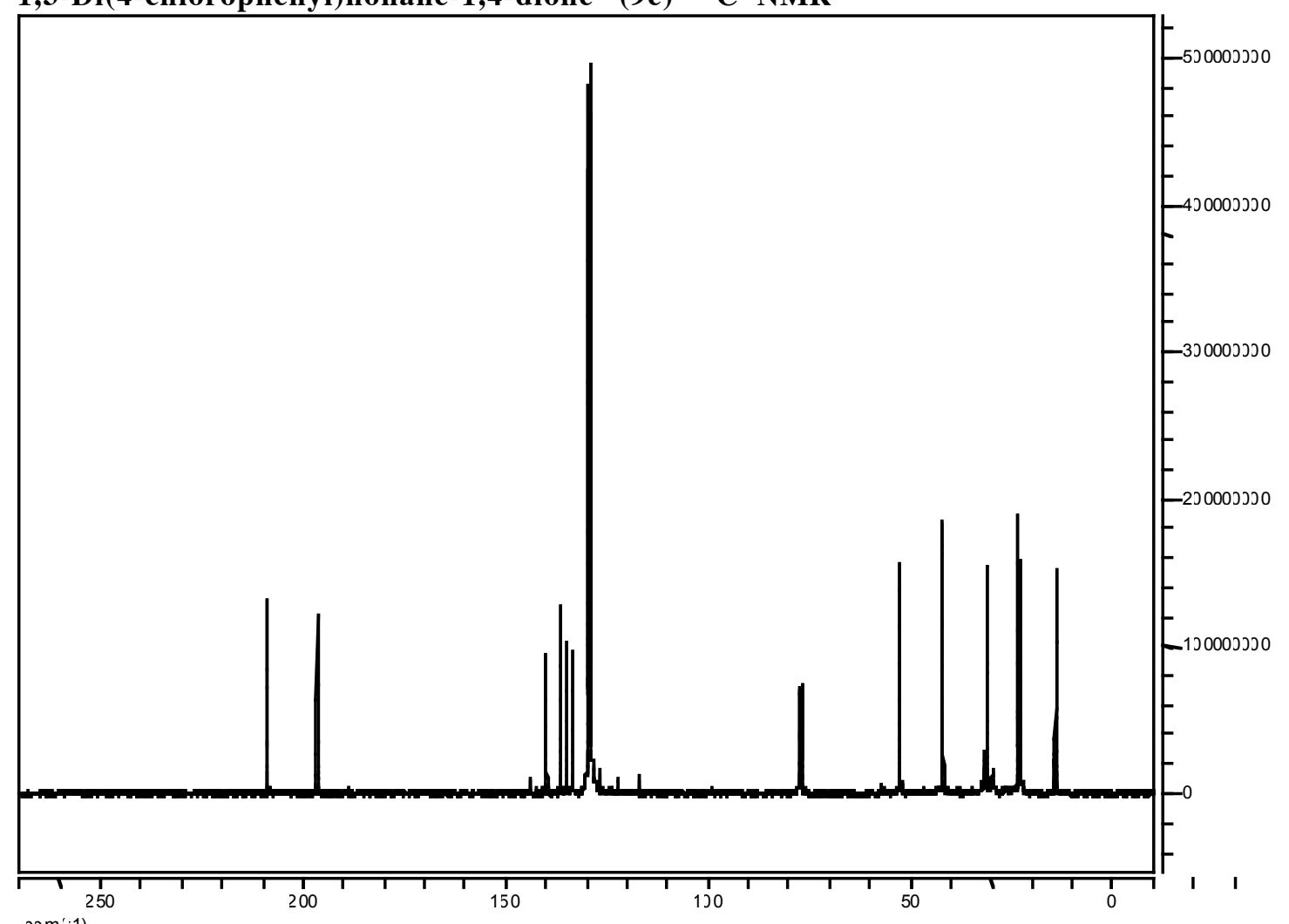

วоm':1) 


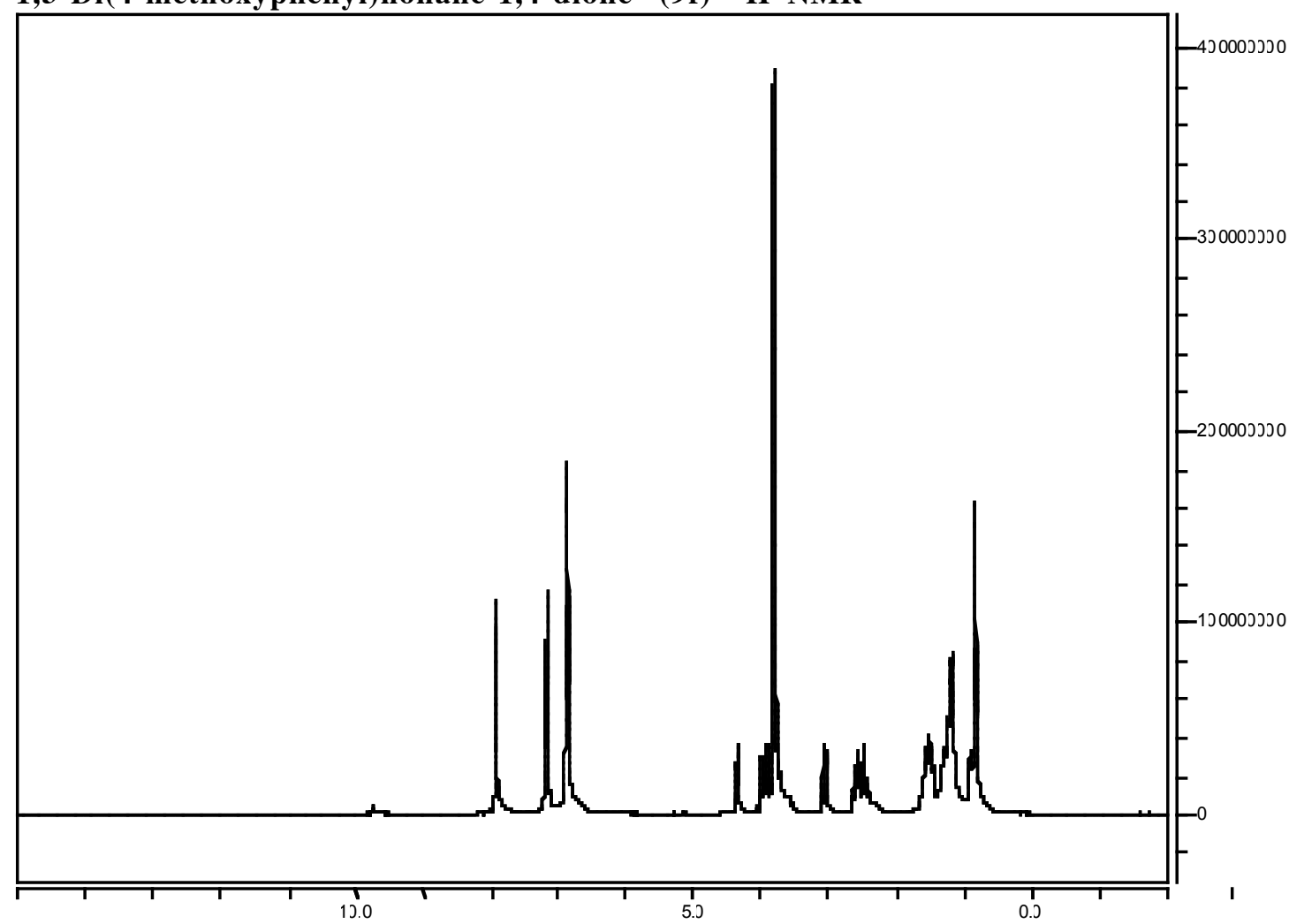

วрm::1)

1,3-Di(4-methoxyphenyl)nonane-1,4-dione (9f) ${ }^{13} \mathrm{C}$ NMR

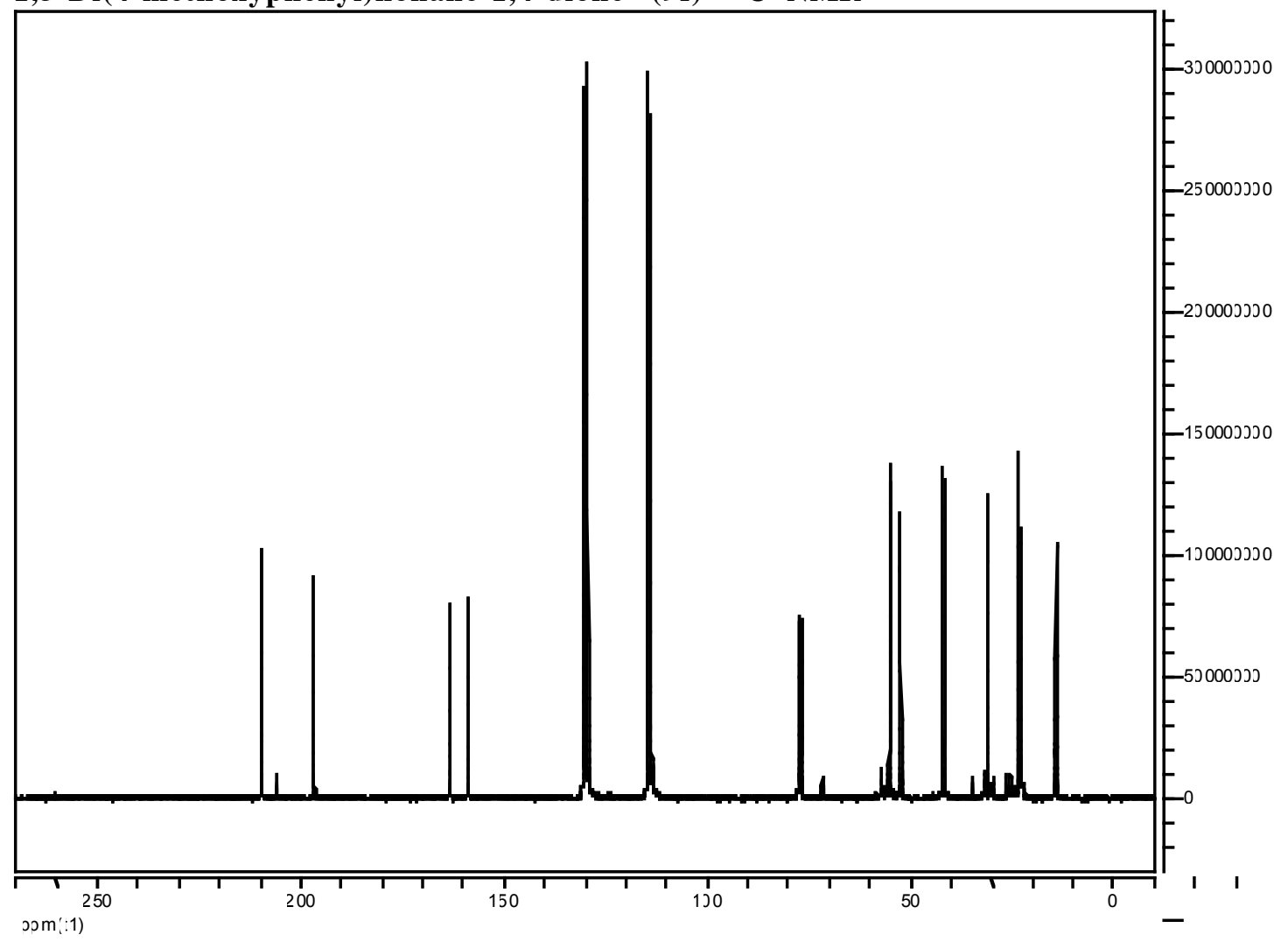

